\title{
Decision fusion with multiple spatial supports by conditional random fields
}

\author{
Devis Tuia, Senior Member, IEEE, Michele Volpi, Member, IEEE, Gabriele \\ Moser, Senior Member, IEEE
}

\begin{abstract}
This is the pre-acceptance version, to read the final version published in the IEEE Transactions on Geoscience and Remote Sensing, please go to: 10.1109/TGRS.2018.2797316 Classification of remotely sensed images into land cover or land use is highly dependent on geographical information at at least two levels. First, land cover classes are observed in a spatially smooth domain separated by sharp region boundaries. Second, land classes and observation scale are also tightly intertwined: they tend to be consistent within areas of homogeneous appearance, or regions, in the sense that all pixels within a roof should be classified as roof, independently on the spatial support used for the classification.

In this paper we follow these two observations and encode them as priors in an energy minimization framework based on conditional random fields (CRFs), where classification results obtained at pixel and region levels are probabilistically fused. The aim is to enforce the final maps to be consistent not only in their own spatial supports (pixel and region) but also across supports, i.e. by getting the predictions on the pixel lattice and on set of the regions to agree. Tho this end, we define an energy function with three terms: 1) a data term for the individual elements in each support (support-specific nodes), 2) spatial regularization terms in a neighborhood for each of the supports (support-specific edges), 3) a regularization term between individual pixels and the region containing each of them (inter-supports edges). We utilize these priors in a unified energy minimization problem that can be optimized by standard solvers. The proposed $2 \mathrm{~L} 々 \mathrm{CRF}$ model consists of a CRF defined over a bipartite graph, i.e. two interconnected layers within a single graph accounting for inter-lattice connections. $2 \mathrm{~L} \downarrow \mathrm{CRF}$ is tested on two very high resolution datasets involving submetric satellite and subdecimeter aerial data. In
\end{abstract}

DT was with the MultiModal Remote Sensing group, University of Zurich, Switzerland. He is now with the GeoInformation Science and Remote Sensing Laboratory, Wageningen University \& Research, the Netherlands. Email: devis.tuia@wur.nl.

MV is with the MultiModal Remote Sensing group, University of Zurich, Switzerland. Email: michele.volpi@geo.uzh.ch.

GM is with the Department of Electrical, Electronic, Telecommunication Engineering, and Naval Architecture, University of Genoa, Italy. Email: gabriele.moser@unige.it.

Digital Object Identifier 10.1109/TGRS.2018.2797316 
all cases, $2 \mathrm{~L} \downarrow \mathrm{CRF}$ improves the result obtained by the independent base model (either random forests or convolutional neural networks) and by standard CRF models enforcing smoothness in the spatial domain.

\section{Index Terms}

Semantic labeling, Classification, Convolutional neural networks, Conditional random fields, Hierarchical models, Region-based analysis.

\section{INTRODUCTION}

Images with metric to decimetric spatial resolutions are becoming the new standard for very high resolution (VHR) remote sensing: images acquired by new generation satellites, as well as by sensors mounted on aircrafts and drones, allow geometrically precise monitoring of the Earth surface and can lead to breakthroughs in agriculture [1], forestry [2], urban characterization [3], and search-and-rescue [4] tasks.

If the promise of very high resolution images is great, the tasks to be solved also become harder: it is well known that as the spatial resolution increases, so does the complexity of semantic labeling (i.e. tasks aiming at assigning each pixel to a semantic class). This is particularly true because of the increase of the intra-class variance and a parallel decrease of the inter-class variance. On one hand, a single semantic class is generally composed of different materials (e.g. a building class is composed spectrally of several heterogeneous materials found on the roof). On the other hand different classes share the same materials (e.g. vegetation is found in both forests and meadows). To address such ambiguity, researchers in remote sensing have explored the use of spatial context and structure, for which we will now review the main families of solutions.

a) Object-based image analysis: A first branch of research considered the possibility to find a coarser spatial support for analysis - typically an agglomeration of pixels, a region prior to learning a classifier. Also known as Object-Based Image Analysis (OBIA [5]), this set of methods aim at defining a new spatial support, possibly respecting the color gradients of the image, extracting some textural and color features at the region level, and learning a supervised classifier. In computer vision, the task of finding coarser but semantically coherent spatial supports is often referred to as superpixelization [6]-[8], where a set of coherent regions 
are found in the image, most often without semantic meaning nor pretense to enclose a complete object in a single region, but only assuming they contain parts of it and share parts of the borders.

b) Spatial filters: A second vivid research subfield is concerned with defining spatial filters, which are image processing techniques applied to the image bands and aiming at characterizing locally relevant properties of the semantic objects to be labeled. For instance, to encode the property that nearby pixels tend to be of the same class, local convolutions implementing low pass filters can be used, while morphological filters [9] and texture filters [10] are often used to encode more complex local relationships. Recently, powerful descriptors from computer vision such as Fisher vectors [11], [12], bag-of-visual-words [13]-[16] or local binary patterns [17] have been proposed to extract spatial information for remote sensing semantic labeling at VHR. But a recurrent problem is to select the right bands to be filtered, the family of filters and their parameters. These are tasks that can prove to be very complex for a non-specialist and whose failure can lead to very suboptimal models. Recent research addresses this issue by learning the relevant filters in an unconstrained space of filter candidates [18], or to learn further combinations of such filters, therefore reaching, through the hierarchy of filters, higher level concepts closer to the semantic classes [19]. Authors in [20] build very large feature sets containing all the information which is possibly useful to characterize the classification problem and then use a classifier that is robust to unimportant features (i.e. embedding feature selection).

c) Convolutional neural networks: The need to learn the relevant filters and features, instead of pre-defining them by expert knowledge, focused recent research in deep learning as a tool for automatically learning multi-scale, nonlinear, semantically tailored, and problem-specific contextual filters jointly with a classifier. Convolutional neural networks (CNNs [21]) are the perfect tool for solving such task: they learn unconstrained convolutional filters hierarchically. The filters learned in the first level (the first convolutional layer) of the network correspond mostly to local oriented gradients, while those learned at the last convolutional layers have a much wider receptive field (they depend on a wider area of the original images) and, therefore, inform about a larger spatial context, usually semantically more related to the problem to be solved. Since the filters are directly learned by backpropagation, no effort has to be provided for filter engineering; but all effort is moved to the design of the network structure itself (number of layers, type of nonlinearities, spatial pooling, size of filters, etc.). For a comprehensive introduction to CNN building blocks in remote sensing, please see [22].

Thanks to numerous benchmark data released recently, the development of CNN architectures 
for the semantic labeling of VHR aerial images has flourished [23]: the first attempts (e.g. [3]) performed inference in a sliding window fashion, therefore retrieving the complete map pixel by pixel. This way one could use a standard classification architecture to map from a patch to a single label (supposed to represent the pixel on which the patch is centered on) and retrieve the whole test map one patch at a time. Besides being inefficient, this also limits the power of the CNN to encode spatial information. A network learned for dense prediction (i.e. outputting the entire map for each pixel in the patch) implicitly encodes spatial relations between the different classes and their features at different scales, while a network predicting a single label and assigning it to the central pixel will consider each patch centered on each pixel independently.

Dense prediction by $\mathrm{CNN}$, for instance inspired by fully convolutional networks [24], hypercolumns [25] or SegNet [26], was proposed in the field of computer vision to upscale the CNN last layers to the original input resolution and de facto provided an elegant, fully learnable, solution to the problem of dense semantic labeling. As a consequence, it was quickly adopted in remote sensing: in [22], [27], [28], authors propose to use learned deconvolution layers to upsample the activations, while in [29] the activations are simply upsampled by interpolation. In [30], [31], authors stack upsampled activations at multiple scales to train other layers performing dense prediction. Finally, in [32] authors propose a network without spatial poolings, i.e. a network that preserves the spatial resolution of the original data throughout all its components.

But besides the impressive performances reported, most approaches i) still showed some residual class inconsistencies and ii) disregarded explicit object structure that could be easily integrated by considering the superpixelization discussed above or some degree of spatial reasoning (e.g. a building rarely occurs in the middle of a river).

d) Structured prediction: The last family of methods addresses this last point by encoding spatial reasoning via interaction potentials between spatial units. Models such as Markov [33][35] and conditional random fields [36], [37] (MRFs or CRFs) can be used to jointly account for different kinds of prior information about spatial relationships and local likelihoods. It has been used extensively in remote sensing to encode different kinds of assumptions one has about the data, such as local spatial smoothness [38]-[40] or, linearity [41] or 3D arrangements [42]. Owing to the Hammersley-Clifford theorem [33], random field models make it possible to express the posterior joint distribution of the unknown class labels given the observations as a Gibbs distribution. This leads to formulating the maximum a-posteriori (MAP) criterion as the mini- 
mization of an appropriate energy function, modeling conditional relationships across variables. The energy can be defined by a number of factors, generally including local likelihood scores from a (set of) classifier(s) [43] and models of spatial relations across locations and their labels. These relations usually encode the likelihood of a pixel to belong to a class, given the class of its direct neighbors [16], [44], or aim at capturing co-occurrence structures among the classes [20], [45], [46]. In addition, the definition of suitable Markovianity properties on hierarchical graphs, including quad-trees, binary partition trees, or more irregular topologies [47], [48], makes it possible to also formalize multiscale and multiresolution fusion within an MRF/CRF framework. Recent approaches based on random fields have been proposed for the semantic segmentation of data acquired at multiple input resolutions at the same time or in a time series [49], [50]. Finally, higher order random field models exist [51], that can be used to encode higher order relationships between elements forming a suitable group (named clique): in remote sensing, such reasoning was exploited to detect roads [52] or relationships between different types of classes occurring on different cliques, such as land cover and land use [53]. Even if these models allow to encode much more complex relationships, they also involve large computational load to perform inference.

e) Co-segmentation: Our method also shares similarities with the domain of image cosegmentation. Research in this direction is often framed as a weakly supervised task, where labels for semantic segmentation or object detection are not dense over the pixel lattice but only appear as general "presence" or "absence" attributes for a given class. It is also commonly phrased as an unsupervised step, where objects belonging to the same object class in different images have to be clustered together. Co-segmentation aims then at building models able to extract common objects in a series of images under the prior that the same objects are present [54], [55].

In this work, we build on the assumption that same classes are present at the same locations, rather than at different locations with possibly different viewpoints. We treat the class label for a given spatial coordinate as the random variable to be fitted from a set of semantic classes, and we do not additionally fit a binary segmentation masks (foreground background) or bounding boxes while grouping the same classes [54]. This simplification has also been used in [56], where co-segmentation of the multitemporal image pair is driven by the pixelwise difference image.

In this paper we aim at fusing the advantages of these families of techniques by proposing a 


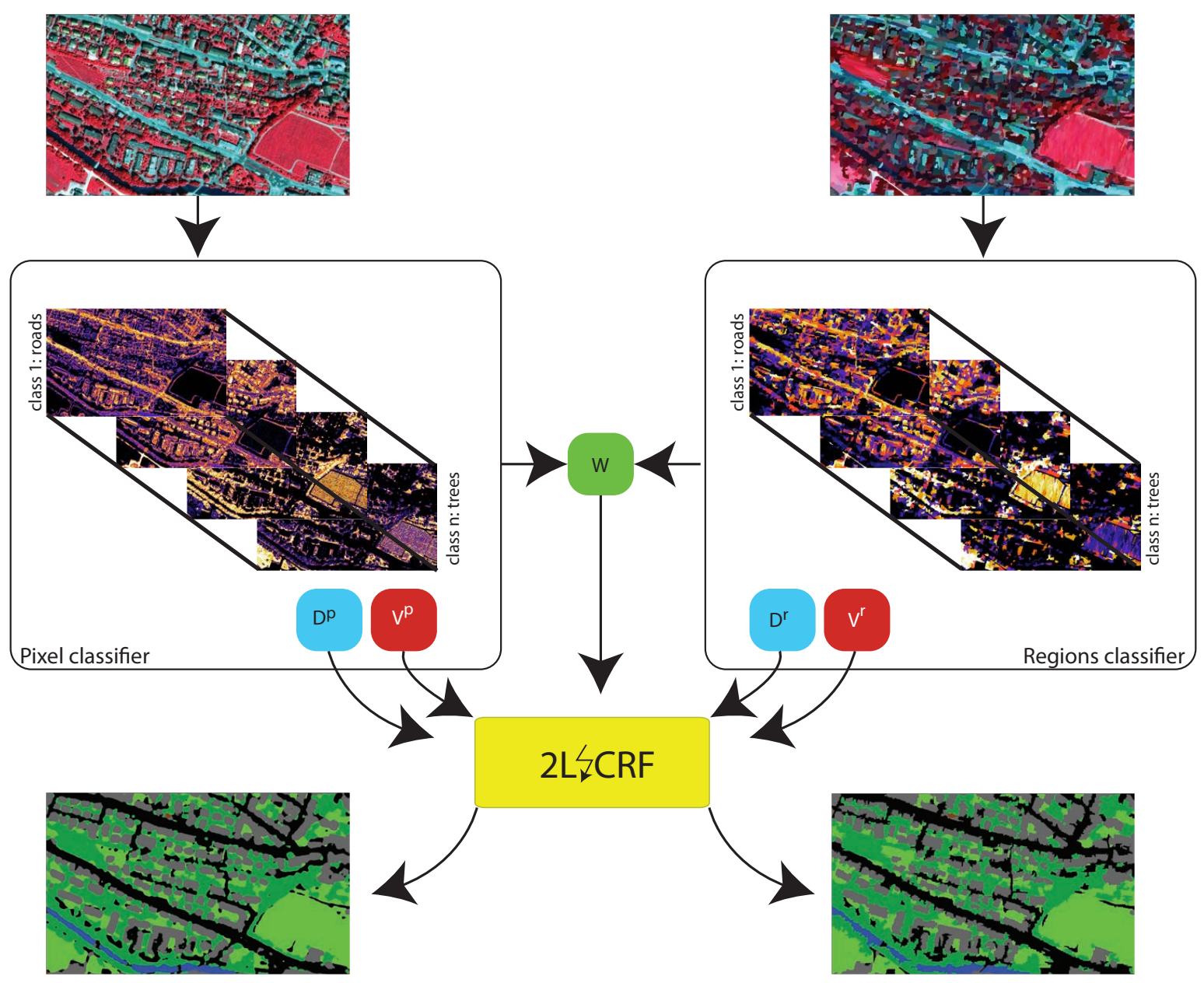

Fig. 1. The proposed 2L $\_$CRF: starting from two models trained on different spatial supports, it uses the posterior probability scores $D$, spatial relations $V$ and hierarchical information $W$ to find the most likely maps (colors and symbols refer to Eq. (6))

model that accommodates different spatial supports (or lattices, connectivity graphs) and encodes spatial reasoning relevant to the problem. We integrate the pixel- and region-based strategies within a multi-scale approach, letting two (or more) spatial supports interact and come to a common decision. The likelihood of each granularity level corresponds to a classification model on a given spatial support. These responses at all levels are fused probabilistically using a bipartite CRF (i.e. a CRF with two interconnected layers [57]). The model, named 2L々CRF (read 'two-layered-lightning-CRF' ${ }^{1}$ ), is sketched in Fig. 1. 2L 2 CRF can use as inputs the

${ }^{1}$ The lightning symbol " $\not$ " is often used in proofs when contradictory statements are involved. Here, it is used on purpose to emphasize that the proposed CRF model is aimed at fusing the two layers, resolving contradictions between their prediction, and "getting pixels and regions" to agree on the final labeling. 
output of any classifier providing a distribution over the labels: they can be obtained by using pre-defined spatial features into standard classifiers or by training CNNs. 2L $\_$CRF performs structured prediction accounting simultaneously for a pixel- and a region-based granularity by encoding 1) spatial structuring via contrast-sensitive pairwise potentials [58] and 2) consistency between the labelings at the pixel and region lattices via an inter-layer smoothness assumption. Contrarily from higher order models such as the PnPotts [51], our conditional random field avoids the multiscale structure of the graph and the use of auxiliary variables by encoding the multiscale problem as a single (flat) graph with interscale connections, thus allowing to use standard and computationally efficient energy minimization solvers. It is more efficient that the iterative formulation of [59] (which is the base of [53]), since it solves both problems within a single energy minimization step.

A preliminary version of this study was previously introduced by the authors in a conference paper [60]. We extend it to the present paper, where we provide an in-depth methodological analysis, a study of the impact of CNN unary scores to the system and results on two datasets, Zurich Summer [16] and the challenging 2015 Data Fusion Contest dataset [3].

In Section $[\mathrm{I}$ we present the formulation of the proposed model, as well as the algorithm used to solve the energy minimization problem. In Section III we present the datasets and the setup of experiments discussed in Section $\mathrm{IV}$. Section $\mathrm{V}$ concludes the paper.

\section{THE PROPOSED 2L $々$ CRF METHOD}

\section{A. Conditional random fields for semantic labeling}

In the framework of semantic labeling, conditional random fields represent a family of probabilistic models allowing to jointly characterize pixelwise class statistics as well as spatial dependencies between the labeling of different neighboring locations. They are among the most used probabilistic graphical models for remote sensing image analysis.

Let us consider a remote sensing image, from which $m$ features have been extracted. The image presents $C$ thematic classes provided with training samples. Let $\mathcal{I}$ be the regular pixel lattice, and $\boldsymbol{x}_{i}$ and $y_{i}$ be the feature vector and the class label of the $i$ th pixel $\left(i \in \mathcal{I} ; \boldsymbol{x}_{i} \in\right.$ $\left.\mathbb{R}^{m} ; y_{i} \in\{1,2, \ldots, C\}\right)$. The CRF approach considers $\boldsymbol{x}_{i}$ and $y_{i}$ as samples from two random fields, i.e. a (generally continuous-valued) random field $\mathcal{X}=\left\{\boldsymbol{x}_{i}\right\}_{i \in \mathcal{I}}$ of feature vectors and a discrete-valued random field $\mathcal{Y}=\left\{y_{i}\right\}_{i \in \mathcal{I}}$ of class labels. Both random fields are supported on the pixel lattice $\mathcal{I}$, on which a neighborhood system $\{\partial i\}_{i \in \mathcal{I}}$ is defined [35]. Common choices 
include the first- and second-order neighborhood systems, in which $\partial i$ is made of the four pixels adjacent to the $i$ th pixel and the eight pixels surrounding it, respectively [40]. With these notations, the random field $\mathcal{Y}$ of the class labels is said to be a CRF if the following posterior Markovianity property holds:

$$
P\left(y_{i} \mid y_{j}, j \neq i, \mathcal{X}\right)=P\left(y_{i} \mid y_{j}, j \in \partial i, \mathcal{X}\right)
$$

and if the (global, imagewise) posterior distribution $P(\mathcal{Y} \mid \mathcal{X})$ is strictly positive [37], [40]. Condition (1) means that the labels are spatially Markovian when conditioned to the random field of the feature vectors. This property makes it possible to express the posterior distribution as $P(\mathcal{Y} \mid \mathcal{X}) \propto \exp [-U(\mathcal{Y} \mid \mathcal{X})]$, where the energy $U(\mathcal{Y} \mid \mathcal{X})$ is defined according to the neighborhood system [33], [40]. Minimizing such energy corresponds to the maximum a posteriori (MAP) criterion. For a system with only pairwise nonzero clique potentials, such energy can be written as [40]:

$$
U(\mathcal{Y} \mid \mathcal{X})=\sum_{i \in \mathcal{I}} D_{i}\left(y_{i} \mid \mathcal{X}\right)+\lambda \sum_{i \in \mathcal{I}} \sum_{j \in \partial i} V_{i j}\left(y_{i}, y_{j} \mid \mathcal{X}\right)
$$

where $D_{i}\left(y_{i} \mid \mathcal{X}\right)$, named unary or association potential, is related to the statistics of each individual label given the feature random field. $V_{i j}\left(y_{i}, y_{j} \mid \mathcal{X}\right)$, named pairwise or interaction potential, encodes the spatial relations among the labels of neighboring pixels $(i \in \mathcal{I}, j \in \partial i$ ). $\lambda$ is a positive parameter that tunes the tradeoff between the unary and pairwise terms. The possibility to characterize the desired spatial interactions by defining suitable pairwise potentials, tailored to the application considered, and the availability of computationally efficient energy minimization algorithms (see also Section II-D) make CRF modeling a powerful and flexible approach to structured prediction in remote sensing image analysis [38], [39].

\section{B. Overview and methodological assumptions of $2 L_{\downarrow} C R F$}

The key idea of the $2 \mathrm{~L} \& \mathrm{CRF}$ method is to benefit from both pixelwise and region-based image representations by introducing a novel model that connects two CRFs, defined on the pixel lattice and on a segmentation result, to perform structured prediction at both levels simultaneously. Given a VHR image, a segmentation method is first applied to identify a set of homogeneous regions. An arbitrary segmentation technique can be used within $2 \mathrm{~L}_{\downarrow} \mathrm{CRF}$, provided that the resulting segments are not particularly coarse.

We denote explicitly with a superscript " $p$ " the quantities introduced in the previous section at the granularity of individual pixels $\left(\mathcal{I}^{p}, \partial^{p} i, \mathcal{X}^{p}, \mathcal{Y}^{p}\right.$, etc.). Let $\mathcal{I}^{r}$ be the set of regions resulting 
from segmentation, $\boldsymbol{x}_{k}^{r}$ be an $n$-dimensional feature vector extracted from the image data of the $k$ th region, and $y_{k}^{r}$ be the class label of the same region $\left(\boldsymbol{x}_{k}^{r} \in \mathbb{R}^{n} ; y_{k}^{r} \in\{1,2, \ldots, C\} ; k \in \mathcal{I}^{r}\right)$. This is equivalent to introducing a second pair of random fields $\mathcal{X}^{r}=\left\{\boldsymbol{x}_{k}^{r}\right\}_{k \in \mathcal{I}^{r}}$ and $\mathcal{Y}^{r}=$ $\left\{y_{k}^{r}\right\}_{k \in \mathcal{I}^{r}}$ that collect feature vectors and labels at the granularity of regions. The proposed method formalizes the relation between labels and feature vectors at each granularity layer as a $\mathrm{CRF}$, and merges these two CRFs into a unique energy (see Section II-C).

From the probabilistic graphical modeling viewpoint, this means using a bipartite graph to combine the two layers and generate a unique labeling at the spatial resolution of the pixel lattice. In the language of data fusion, the method fuses the information associated with pixelwise statistics and with two sources of spatial information, i.e. local neighborhoods and region-based reasoning. From a computational perspective, the resulting energy is also equivalent to a casespecific single-layer model on a planar graph - a property that makes it possible to use timeefficient algorithms to numerically address the energy minimization task (see Section II-D).

More precisely, $2 \mathrm{~L} \downarrow \mathrm{CRF}$ is based on the following methodological assumptions:

1) In addition to the neighborhood system $\left\{\partial^{p} i\right\}_{i \in \mathcal{I}^{p}}$ on the pixel lattice, a neighborhood system $\left\{\partial^{r} j\right\}_{j \in \mathcal{I}^{r}}$ is also defined on the set of regions.

2) The random field $\mathcal{Y}^{s}$ of the labels at each granularity layer $s \in\{p, r\}$, given the corresponding random field $\mathcal{X}^{s}$ of the feature vectors, is a CRF with up to pairwise nonzero clique potentials.

3) The following conditional independence assumption holds:

$$
f\left(\mathcal{X}^{p}, \mathcal{X}^{r} \mid \mathcal{Y}^{p}, \mathcal{Y}^{r}\right)=f\left(\mathcal{X}^{p} \mid \mathcal{Y}^{p}\right) f\left(\mathcal{X}^{r} \mid \mathcal{Y}^{r}\right)
$$

where $f\left(\mathcal{X}^{p}, \mathcal{X}^{r} \mid \mathcal{Y}^{p}, \mathcal{Y}^{r}\right)$ is the joint probability density function (PDF) of all feature vectors conditioned to all labels in both granularity layers, and $f\left(\mathcal{X}^{p} \mid \mathcal{Y}^{p}\right)$ and $f\left(\mathcal{X}^{r} \mid \mathcal{Y}^{r}\right)$ are the class-conditional PDFs associated with the two layers separately.

In particular, a second order neighborhood is used on the pixel lattice, and two regions are considered to be neighbors (see Assumption 1) if they share some common boundary (see Fig. 2). Equation (3) indicates that the statistics of the features in the two layers are modeled as independent when conditioned to the labels of each layer (see Assumption 3). From a modeling viewpoint, this condition allows the class-conditional statistics within each layer to be characterized separately. This conditional independence assumption ensures mathematical 


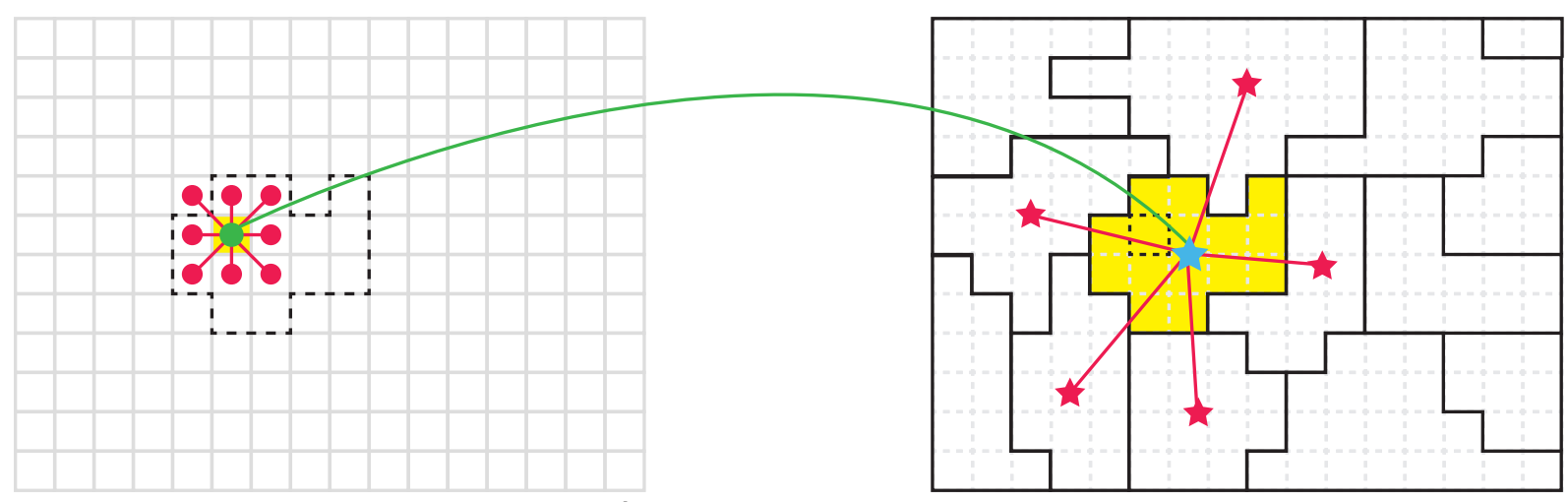

Pixels

Regions

Fig. 2. General idea of the $2 \mathrm{~L} \downarrow \mathrm{CRF}$ structured prediction model used to connect the pixel lattice and the set of regions. Red links represent the pairwise contrast-sensitive potentials ([B] terms in 6 between pixels (represented by circles) or region centroids (represented by stars). The green link represents the cross-layer potential relating a region and each pixel included in that region ([C] term in (6)). The locations of the considered pixel and region in the other layer are highlighted with dashed black lines.

tractability and is often accepted in MRF-based (and more generally Bayesian) approaches to spatial-contextual, multisource, or multitemporal classification [35], [40], [43], [61], [62].

\section{The proposed two-layers model}

To apply the MAP decision rule over pixels and regions simultaneously, we consider the joint posterior distribution of the combined random field $\mathcal{Y}=\left(\mathcal{Y}^{p}, \mathcal{Y}^{r}\right)$ of pixel and region labels, given the combined random field $\mathcal{X}=\left(\mathcal{X}^{p}, \mathcal{X}^{r}\right)$ of all feature vectors on both layers. Based on Assumptions 1-2-3, we prove that this joint posterior $P(\mathcal{Y} \mid \mathcal{X})$ can be expressed as a function of i) two separate contributions associated with the marginal posteriors $P\left(\mathcal{Y}^{p} \mid \mathcal{X}^{p}\right)$ and $P\left(\mathcal{Y}^{r} \mid \mathcal{X}^{r}\right)$ of the two granularity layers, which are modeled as CRFs (see Assumption 2); and ii) a cross-layer term that is related to the dependence between $\mathcal{Y}^{p}$ and $\mathcal{Y}^{r}$ and provides a prior on the desired relations between region labels and pixel labels.

The Bayes theorem implies that:

$$
\begin{aligned}
P(\mathcal{Y} \mid \mathcal{X}) & =P\left(\mathcal{Y}^{p}, \mathcal{Y}^{r} \mid \mathcal{X}^{p}, \mathcal{X}^{r}\right) \propto \\
& \propto f\left(\mathcal{X}^{p}, \mathcal{X}^{r} \mid \mathcal{Y}^{p}, \mathcal{Y}^{r}\right) \cdot P\left(\mathcal{Y}^{p}, \mathcal{Y}^{r}\right)
\end{aligned}
$$

where the proportionality factor depends on the feature vector fields $\mathcal{X}^{p}$ and $\mathcal{X}^{r}$, but not on the label fields $\mathcal{Y}^{p}$ and $\mathcal{Y}^{r}$, hence it does not influence MAP decisions. Owing to Assumption 3 and 
using again the Bayes theorem, we obtain:

$$
\begin{aligned}
P(\mathcal{Y} \mid \mathcal{X}) & \propto P\left(\mathcal{Y}^{p}, \mathcal{Y}^{r}\right) \prod_{s \in\{p, r\}} f\left(\mathcal{X}^{s} \mid \mathcal{Y}^{s}\right) \propto \\
& \propto P\left(\mathcal{Y}^{p}, \mathcal{Y}^{r}\right) \prod_{s \in\{p, r\}} \frac{P\left(\mathcal{Y}^{s} \mid \mathcal{X}^{s}\right)}{P\left(\mathcal{Y}^{s}\right)}= \\
& =\frac{P\left(\mathcal{Y}^{p}, \mathcal{Y}^{r}\right)}{P\left(\mathcal{Y}^{p}\right) P\left(\mathcal{Y}^{r}\right)} \cdot \prod_{s \in\{p, r\}} P\left(\mathcal{Y}^{s} \mid \mathcal{X}^{s}\right),
\end{aligned}
$$

where the proportionality factor depends again only on $\mathcal{X}^{p}$ and $\mathcal{X}^{r}$. Equation (5) provides the desired factorization of the joint posterior.

Accordingly, the MAP rule is expressed as the minimization of the following energy function with respect to $\mathcal{Y}$ :

$$
\begin{aligned}
U(\mathcal{Y} \mid \mathcal{X}) & =\sum_{s \in\{p, r\}} \sum_{i \in \mathcal{I}_{s}} \underbrace{D^{s}\left(y_{i}^{s} \mid \mathcal{X}^{s}\right)}_{[\mathbf{A}]}+ \\
& +\lambda \sum_{s \in\{p, r\}} \sum_{i \in \mathcal{I}_{s}} \sum_{j \in \partial^{s} i} \underbrace{V^{s}\left(y_{i}^{s}, y_{j}^{s} \mid \mathcal{X}^{s}\right)}_{[\mathbf{B}]}+ \\
& +\mu \underbrace{W\left(\mathcal{Y}^{p}, \mathcal{Y}^{r}\right)}_{[\mathbf{C}]},
\end{aligned}
$$

where $\lambda$ and $\mu$ are positive weight parameters and the three terms highlighted have the following meaning:

[A] This is the unary potential contributing to the CRF model for each single-layer posterior $P\left(\mathcal{Y}^{s} \mid \mathcal{X}^{s}\right)$ in (5). As a customary choice in many MRF- and CRF-based methods [16], [40], [45], we compute it as $\left(i \in \mathcal{I}^{s}\right)$ :

$$
D^{s}\left(y_{i}^{s} \mid \mathcal{X}^{s}\right)=-\ln \hat{P}^{s}\left(y_{i}^{s} \mid \boldsymbol{x}_{i}^{s}\right)
$$

where $\hat{P}^{s}\left(y_{i}^{s} \mid \boldsymbol{x}_{i}^{s}\right)$ is an estimate of the element-wise posterior probability of a pixel or region. It can be computed by training, at layer $s$, a classifier that provides a probabilistic output (e.g., a parametric or non-parametric Bayesian classifier [63], a random forest [64], a CNN [21], or the postprocessing of the output of a support vector machine [65] by algorithms such as [66]).

Note that this model for the unary potential in $2 \mathrm{~L} \downarrow \mathrm{CRF}$ is intrinsically homogeneous, as reflected by the absence of the subscript " $i$ " in (6) and (7), as compared to (2) (i.e., $D^{s}(\cdot)$ instead of $D_{i}^{s}(\cdot)$ ) [40]. This homogeneity in the unary potential is not considered a critical restriction because the region-based analysis incorporates local spatial adaptivity per se. 
[B] This energy contribution is a pairwise term that favors spatial smoothness at each granularity level (see the red line in Fig. 2). It completes the CRF model for $P\left(\mathcal{Y}^{s} \mid \mathcal{X}^{s}\right)$ along with the aforementioned unary term [A]. $V^{s}\left(y_{i}^{s}, y_{j}^{s} \mid \mathcal{X}^{s}\right)$ is defined as a contrast-sensitive potential that extends the classical Potts MRF model in order to penalize that different classes are predicted for neighboring pixels or regions with similar feature vectors [58]. In 2L $\{\mathrm{CRF}$, contrast sensitivity is modeled using a Gaussian kernel $K(\cdot)\left(i \in \mathcal{I}^{s}, j \in \partial^{s} i\right)$ :

$$
V^{s}\left(y_{i}^{s}, y_{j}^{s} \mid \mathcal{X}^{s}\right)=\left[1-\delta\left(y_{i}^{s}, y_{j}^{s}\right)\right] K\left(\boldsymbol{x}_{i}^{s}, \boldsymbol{x}_{j}^{s}\right)
$$

where $\delta(\cdot)$ is the Kronecker symbol (i.e., $\delta(a, b)=1$ for $a=b$ and $\delta(a, b)=0$ otherwise). For instance, if two neighboring pixels have identical feature vectors (hence $K\left(\boldsymbol{x}_{i}^{p}, \boldsymbol{x}_{j}^{p}\right)=1$ ) and are predicted in different classes, then the maximum penalty is applied. On the contrary, if their feature vectors differ substantially (thus $K\left(\boldsymbol{x}_{i}^{p}, \boldsymbol{x}_{j}^{p}\right) \simeq 0$ ), then assigning the two pixels to different classes is not (or very slightly) penalized. The same comment holds in the case of regions. The previous remark on homogeneity holds in this case (8) as well. Note that the same parameter $\lambda$ is used in (6) to weigh both $V^{p}(\cdot)$ and $V^{r}(\cdot)$. This is consistent with the idea of giving both granularity layers the same relevance in the labeling process. Nevertheless, extending (6) with different weight parameters for the pixel and region granularities is straightforward.

[C] This energy term is a cross-layer pairwise contribution that favors consistency between the labelings at the two granularity levels (see the green line in Fig. 2) and corresponds, up to additive or positive multiplicative constants, to $-\ln \left\{P\left(\mathcal{Y}^{p}, \mathcal{Y}^{r}\right) /\left[P\left(\mathcal{Y}^{p}\right) P\left(\mathcal{Y}^{r}\right)\right]\right\}$. This term is related to the joint prior $P\left(\mathcal{Y}^{p}, \mathcal{Y}^{r}\right)$ and provides a measure of the dependence between the fields $\mathcal{Y}^{p}$ and $\mathcal{Y}^{r}$. The rationale of [C] is to encode the desired agreement between pixelwise and region-based results. This behavior is favored in the proposed method by using a Potts-like formulation:

$$
W\left(\mathcal{Y}^{p}, \mathcal{Y}^{r}\right)=\sum_{i \in \mathcal{I}^{p}}\left[1-\delta\left(y_{i}^{p}, y_{i^{\uparrow}}^{r}\right)\right]
$$

where $i^{\uparrow}$ indicates the region to which the $i$ th pixel belongs, i.e. $y_{i}^{p}$ and $y_{i \uparrow}^{r}$ are the labels of this pixel at the pixel and region levels, respectively $\left(i \in \mathcal{I}^{p}\right)$. Indeed, 9 contributes a penalty for each pixel for which the classes predicted at the two levels differ. 


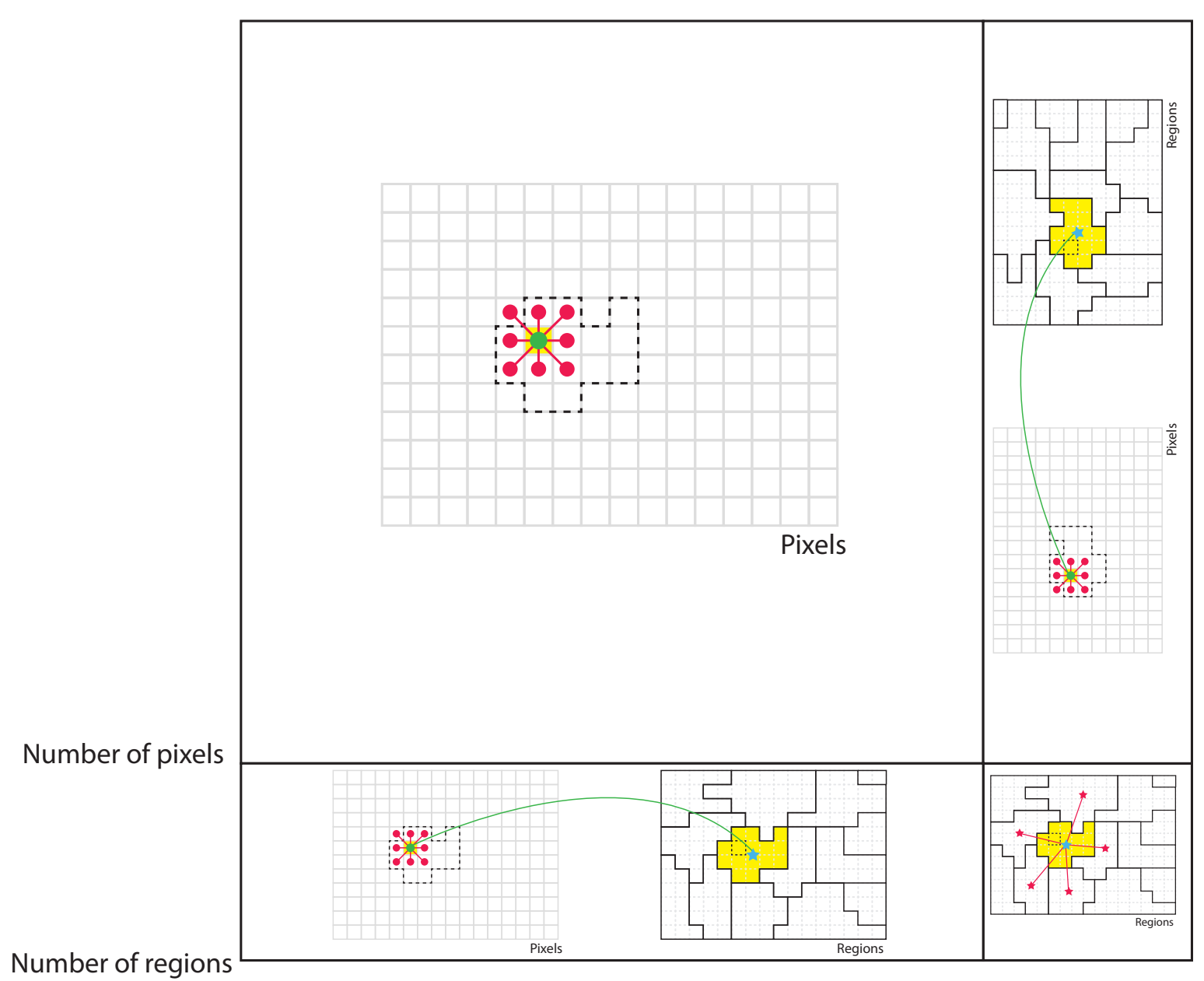

Fig. 3. Intuition behind the graph structure used in the dual layer.

More generally, formulating the decision fusion over multiple supports as the minimization of energy (6) can also be interpreted as a variational representation, especially in relation to the belief-propagation-type method that is used to address it (see Section [II-D] [37], [67].

\section{Flattening the two layers into a single graph and minimizing the energy}

The problem of the minimization of CRF energy functions such as (2) is generally a complex combinatorial problem. Nevertheless, in addition to consolidated stochastic optimization approaches such as simulated annealing [33], computationally efficient graph-theoretic algorithms have become very prominent during the past decade [40], [68]. They include graph cut algorithms, which make use of a min-flow/max-cut reformulation of the minimum energy problem [58], and belief propagation-type algorithms, which build on the idea of exchanging messages between 
neighboring elements to progressively reduce the energy [69]. These techniques have been found successful to minimize energies that are supported on a pixel lattice (as in (2)) or even on more general graphs [68]. On one hand, in the case of the proposed method, two layers with distinct supports are involved in the minimization task simultaneously, a situation that does not allow applying these methods directly. On the other hand, we show here that $2 \mathrm{~L} \not \mathrm{CRF}$ can also be equivalently reformulated on a unique planar graph, thus making it possible to use the aforementioned energy minimization methods appropriately.

The idea is to combine the two granularity layers into a single undirected graph $\mathcal{G}=(\mathcal{V}, \mathcal{E})$, in which both the spatial [B] and the cross-layer [C] pairwise interactions are reorganized. As shown in Fig. 3, the set of nodes is $\mathcal{V}=\mathcal{I}^{p} \cup \mathcal{I}^{r}$ (i.e. it includes all pixels and regions), and an edge exists between two nodes $u$ and $v$, i.e., $(u, v) \in \mathcal{E}$, if and only if one of the following conditions holds $(u, v \in \mathcal{V})$ :

- $u$ and $v$ are neighboring pixels, i.e. $u \in \mathcal{I}^{p}, v \in \partial^{p} u$;

- $u$ and $v$ are neighboring regions, i.e. $u \in \mathcal{I}^{r}, v \in \partial^{r} u$;

- $u$ is a pixel and $v$ is the region that includes $u$, i.e. $u \in \mathcal{I}^{p}$ and $v=u^{\uparrow}$, or vice versa.

The random fields $\mathcal{X}^{p}, \mathcal{X}^{r}, \mathcal{Y}^{p}$, and $\mathcal{Y}^{r}$ on the pixel lattice and on the set of regions can be rearranged on the graph $\mathcal{G}$ in a straightforward way (see Fig. 3). Denoting with a bar the rearranged quantities (e.g., $\overline{\mathcal{Y}}=\left\{\bar{y}_{v}\right\}_{v \in \mathcal{V}}$, where $\bar{y}_{v}$ indicates the label of node $v$ and is either $y_{v}^{p}$ or $y_{v}^{r}$ as $v \in \mathcal{I}^{p}$ or $v \in \mathcal{I}^{r}$, respectively), the energy (6) can be rewritten as:

$$
\bar{U}(\overline{\mathcal{Y}} \mid \overline{\mathcal{X}})=\sum_{v \in \mathcal{V}} \bar{D}_{v}\left(\bar{y}_{v} \mid \overline{\mathcal{X}}\right)+\sum_{(u, v) \in \mathcal{E}} \bar{V}_{u v}\left(\bar{y}_{u}, \bar{y}_{v} \mid \overline{\mathcal{X}}\right)
$$

where the unary potential is:

$$
\bar{D}_{v}\left(\bar{y}_{v} \mid \overline{\mathcal{X}}\right)= \begin{cases}D^{p}\left(y_{v}^{p} \mid \mathcal{X}^{p}\right) & \text { if } v \in \mathcal{I}^{p} \\ D^{r}\left(y_{v}^{r} \mid \mathcal{X}^{r}\right) & \text { if } v \in \mathcal{I}^{r}\end{cases}
$$

and the pairwise potential is:

$$
\begin{aligned}
& \bar{V}_{u v}\left(\bar{y}_{u}, \bar{y}_{v} \mid \overline{\mathcal{X}}\right)= \\
& = \begin{cases}\lambda\left[1-\delta\left(\bar{y}_{u}, \bar{y}_{v}\right)\right] K\left(\overline{\boldsymbol{x}}_{u}, \overline{\boldsymbol{x}}_{v}\right) & \text { if }\left(u \in \mathcal{I}^{p} \text { and } v \in \partial^{p} u\right) \\
\mu\left[1-\delta\left(\bar{y}_{u}, \bar{y}_{v}\right)\right] & \text { or }\left(u \in \mathcal{I}^{r} \text { and } v \in \partial^{r} u\right)\end{cases} \\
& \text { if }\left(u \in \mathcal{I}^{p} \text { and } v=u^{\uparrow}\right)
\end{aligned}
$$


Note that, although the unary and pairwise potentials in (7) and (8) are homogeneous, the potentials $\bar{D}_{v}(\cdot)$ and $\bar{V}_{u v}(\cdot)$ on the flattened planar graph $\mathcal{G}$ are only piecewise homogeneous.

To minimize the resulting energy $\bar{U}(\overline{\mathcal{Y}} \mid \overline{\mathcal{X}})$ with respect to $\overline{\mathcal{Y}}$ on the graph $\mathcal{G}$ (i.e. with respect to $\mathcal{Y}^{p}$ and $\mathcal{Y}^{r}$ simultaneously), the sequential tree re-weighted message passing (TRW-S) algorithm is used in $2 \mathrm{~L} \downarrow \mathrm{CRF}$. This algorithm integrates the belief propagation approach and the construction of appropriate spanning trees on the considered graph, and makes use of a specific sequential formulation to favor a convergent behavior (details can be found in [69]).

\section{DATA AND SETUP}

In this Section, we introduce and discuss the datasets used for the experiments. We also detail the setup of said experiments.

\section{A. Data}

The performance of the proposed $2 \mathrm{~L} \downarrow \mathrm{CRF}$ is tested using two very-high resolution datasets: the Zurich Summer and the Zeebruges datasets.

- The Zurich Summer Dataset [20]. This dataset is a collection of 20 images from a single large QuickBird (NIR-RGB bands) acquisition of 2002. The images picture different neighborhoods of the city of Zurich, Switzerland. Each image is pansharpened to $0.6 \mathrm{~m}$ resolution and labeled in 8 classes. The labeling is not dense, meaning that some pixels are either unassigned or belonging to unseen classes. False color infrared images and the ground truth for five tiles can be seen in the first two columns of Fig. 6. The dataset can be freely downloaded at https://sites.google.com/site/michelevolpiresearch/data/zurich-dataset.

- Zeebruges, or the Data Fusion Contest 2015 dataset (grss_dfC_2015) [3]. The Image Analysis and Data Fusion Technical Committee of the IEEE Geoscience and Remote Sensing Society organized in 2015 a data processing competition which aimed at 5-centimeter resolution land cover mapping. Both an RGB aerial image $(5 \mathrm{~cm}$ spatial resolution) and a dense lidar point cloud $\left(65 \mathrm{pts} / \mathrm{m}^{2}\right)$ were acquired over the harbor of Zeebruges, Belgium. The data are organized on seven $10,000 \times 10,000$ pixels tiles. We used a downgraded version on $5,000 \times 5,000$ pixels for training the models and upsampled the final prediction using interpolation (the final numerical scores were not significantly affected, but computational efficiency was dramatically improved). All the tiles have been densely annotated in 8 land classes, including land use (buildings, roads) and small objects (vehicles, boats) classes 
Tile 1

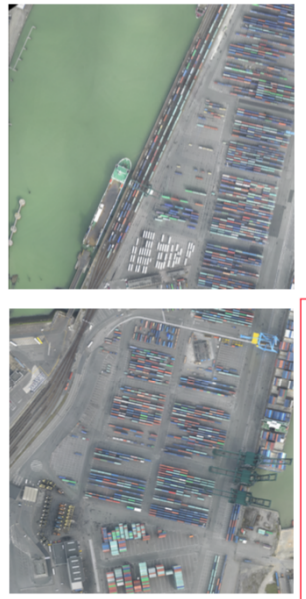

Tile 3

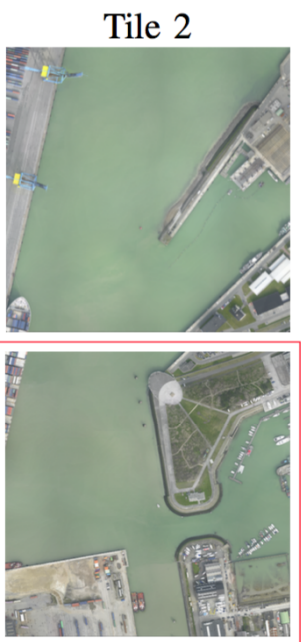

Tile 4

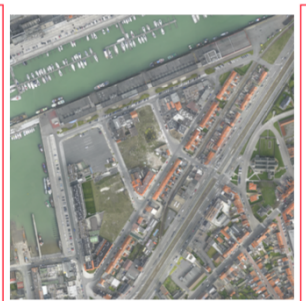

Tile 5

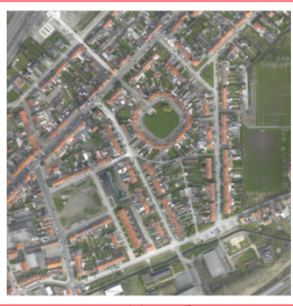

Tile 6

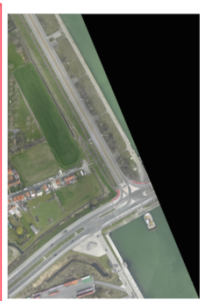

Tile 7

Fig. 4. The seven tiles of the Data Fusion Contest 2015 dataset (RGB) (from [3]). Test tiles are highlighted in red. The data can be freely downloaded from http://www.grss-ieee.org/community/technical-committees/data-fusion/ 2015-ieee-grss-data-fusion-contest/ (Note: in this preprint we had to decrease the graphics resolution. For full resolution, please refer to the published version, or contact the authors).

by the authors of [70]. The seven RGB tiles, as well as the LiDAR normalized DSM are illustrated in Figs. 4 and 5. The ground truth test tiles are presented only in a blurred manner, since they are undisclosed to the participants of the challenge. The data can be obtained from the IEEE GRSS Data and Algorithm Standard Evaluation Website (DASE) http://dase.ticinumaerospace.com//. From DASE, users can download the seven tiles and labels for five tiles. To assess models on the two remaining tiles, we uploaded the classification maps on the DASE server, which automatically computes confusion matrices and accuracy scores. For more information about the data, please refer to [3] and https://www.grss-ieee. org/community/technical-committees/data-fusion/2015-ieee-grss-data-fusion-contest/.

\section{B. Experimental setup}

The experiments presented in the next section aim at showcasing the effectiveness of the proposed $2 \mathrm{~L}_{\downarrow} \mathrm{CRF}$ in different settings using different classifiers. In all experiments, we compare the results obtained using models aware of a single support (i.e. only pixel- or region-based mapping units) with those obtained by models exploiting structure in the spatial domain of the support (in all cases, the CRF of [51]) and with the results of the proposed model, which lets 
Tile 1
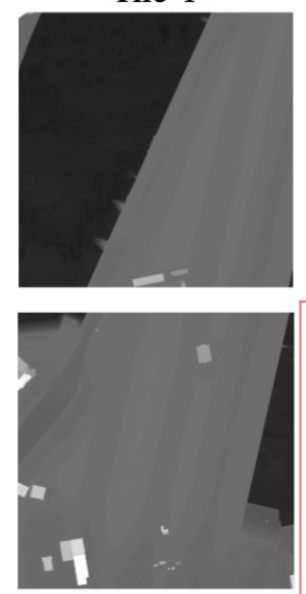

Tile 3
Tile 2
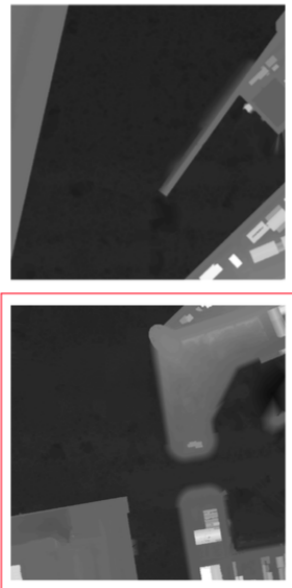

Tile 4

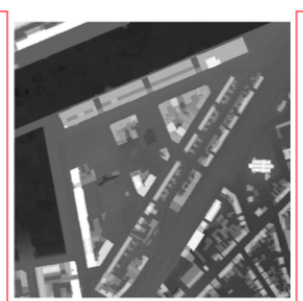

Tile 5

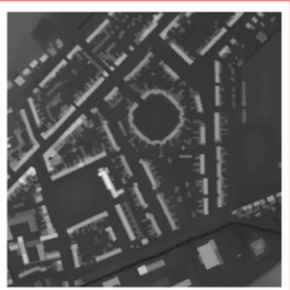

Tile 6

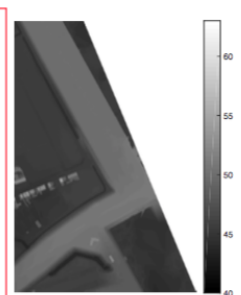

Tile 7

Fig. 5. The seven tiles of the DSM issued from the LiDAR point cloud of the Data Fusion Contest 2015 (from [3]). Test tiles are highlighted in red. The data can be freely downloaded from http://www.grss-ieee.org/community/technical-committees/ data-fusion/2015-ieee-grss-data-fusion-contest/. (Note: in this preprint we had to decrease the graphics resolution. For full resolution, please refer to the published version, or contact the authors).

the two supports interact via the two-layers CRF structure. Below we present the setups used for the two datasets.

1) Zurich summer: We consider two base classifiers: random forests [64] and convolutional neural networks [21] with independent patch-response.

- Random forest $(R F)$. In the case of random forests, we train two separate models, one at the pixel and another at the region level [60]. At the pixel level, we use spectral features (RG-B-NIR raw values, normalized to standard scores), the normalized difference vegetation and water indices (NDVI and NDWI), and contextual features (average over $3 \times 3$ and $5 \times 5$ local windows, computed on the R and NIR bands). Therefore, we have a 10-dimensional input space. Regarding the region level, we first extract regions using the Felzenszwalb and Huttenlocher graph-based algorithm [6]. We used as region descriptors the min, max, average and standard deviation values of the pixel intensities included in each region for the R, G, B, NIR, NDVI, and NDWI channels. The input space of the region classifier is therefore 24-dimensional. To train the models, we derived a region ground truth from the available pixelwise training map by assigning the majority class found within each region.

- Convolutional neural network. In the case of CNNs, we opted for a classical image classi- 
fication architecture, i.e. an architecture that predicts a single label per patch (in our case, of size $65 \times 65$ pixels) and predicts the final map using a sliding window approach, as in [3]. The reasoning behind it is that, since the ground truth is not dense, spatial structures become less explicit to be learned, and complex optimization schemes (as the one in [16]), which are not trivial to stack on top of a dense prediction CNN, should be involved to learn them properly. Specifically, we use four convolutional blocks, the first two with $5 \times 5$ filters (expressed as two consecutive $3 \times 3$ filters, as proposed in [71]) and the other two with plain $3 \times 3$ filters. Each filter is followed by a ReLU nonlinearity and by a spatial pooling halving the spatial extent of the activations. The fourth block is followed by a fully connected layer outputting a 256-dimensional vector per patch. This vector is then used to predict the class conditional probabilities with a soft-max classifier. A dropout [72] rate of $50 \%$ is used on the fully connected layer to reduce the risk of overfitting the training data. Given the computational efforts that would have been involved, we did not train a specific region-based model, but rather averaged and re-normalized the class probabilities per region and used them as scores for the region. The results were not affected by using the averaged CNN pixelwise class probabilities instead of training a specific model for the region lattice (be it a CNN or the RF model presented above).

In both cases, the neighborhood systems used are a second order ( 8 direct neighbors) at the pixel level and the set of regions sharing a common boundary with the region under consideration at the region level.

We use images ID 1-15 (out of the 20 images composing the dataset) to train the models and images 16-20 to test its generalization. Accuracy figures are provided per image and averaged over the test set, for both the pixel and region levels. For the case of random forest we trained the pixel-level classifier with $0.01 \%$ of the available labeled pixels, with a selection stratified by class (corresponding to 122, 658 pixels pooled from all the training images). At the region level, the second RF classifier used all the available 34,020 labeled regions. The increase of training set size at pixel level with respect to [60] is due to the intention of providing a better numerical comparison against the $\mathrm{CNN}$ model, for which 10,000 patches are used for training, randomly selected, and re-sampled every 10 training epochs. Since we run 150 epochs of the model, the CNN actually saw roughly 150,000 patches during the whole training, whose diversity was also increased by data augmentation, i.e. random flips and rotations at each 10 epochs resampling. 
The CNN was trained by stochastic gradient descent with momentum (0.9), with a weight decay of 0.001 and a constant learning rate of $10^{-3}$.

2) Zeebruges: In the case of Zeebruges, we opted for the CNN case only, as Random Forest do not perform nearly as accurate. In [3], two main observations were made: CNN outperformed other nonlinear methods (such as support vector machines) and a fully-trained model provided the most accurate solution on this dataset. We also opted for a dense prediction architecture: for this dataset, we have a dense ground truth [70] and therefore spatial relationships can be explicitly learned. Following these two reasons, we used the CNN in [31], whose architecture follows the concept of hypercolumns [25], but with added equivariance to rotation. The CNN is based on a set of convolutional blocks with spatial pooling and all the activations at each level are upsampled to the original image resolution, stacked and then fed into a second block employing this multiscale descriptions to map to class likelihoods. In our case, the convolutional blocks are RotEqNet layers enforcing rotation equivariance of the final prediction (see [73] for details). There are six such blocks, learning respectively $[14,14,21,28,28,28]$ filters of size $7 \times 7$ each. After concatenation of the activations, the $\mathrm{CNN}$ has three $1 \times 1$ convolution layers with 350, 350, and 6 (the number of classes) neurons, and a softmax normalization. The model was trained for 34 iterations with decreasing weight decay (from $10^{-1}$ to $4 \cdot 10^{-3}$ ) and learning rates (from $10^{-2}$ to $4 \cdot 10^{-4}$ ).

Finally, for both datasets hyperparameters $\mu$ and $\lambda$ were set by crossvalidation and the $\sigma$ parameter of the kernel in the contrast sensitive term (Eq. 8) was set as half the Euclidean features distance between samples.

\section{EXPERIMENTAL RESULTS}

In this section we present the results obtained on the Zurich Summer and Zeebruges datasets.

\section{A. Zurich Summer dataset}

For the Zurich Summer dataset, we first discuss results obtained by the approach considering random forests with contextual features and then compare it against the results obtained by the CNN base classifier.

Random forests. Table $\Pi$ shows the numeric scores obtained using random forest. Results are in line with those reported in [60]. The slight increases in accuracies are due to the larger training set used to train the pixel-based classifier and are consistent across the experiments. When using 
TABLE I

ZURICH SUMMER DATASET: NUMERICAL RESULTS ON THE FIVE TEST TILES. EACH PAIR OF COLUMNS ILLUSTRATES THE CHANGE OF PERFORMANCE BETWEEN THE UNARIES (RANDOM FOREST) AND THE SPATIAL MODEL USING THEM AS A BASE PROBABILISTIC INPUT IN THE UNARY POTENTIAL. THE CRF IS BASED ON [58].

\begin{tabular}{|c|c|c|c|c|c|c|c|c|c|c|c|c|c|c|}
\hline \multirow{3}{*}{$\begin{array}{c}\text { Tile } \\
\# \\
\end{array}$} & \multicolumn{5}{|c|}{ Ov. Accuracy (OA) } & \multicolumn{5}{|c|}{ Kappa $(\kappa)$} & \multicolumn{4}{|c|}{ Av. Accuracy (AA) } \\
\hline & \multicolumn{3}{|c|}{ Pixels } & \multicolumn{2}{|r|}{ Regions } & \multicolumn{3}{|c|}{ Pixels } & \multicolumn{2}{|r|}{ Regions } & \multicolumn{2}{|r|}{\begin{tabular}{l|l} 
Pixels \\
\end{tabular}} & \multicolumn{2}{|c|}{ Regions } \\
\hline & $\mathrm{RF}$ & CRF & $2 \mathrm{~L} 々 \mathrm{CRF}$ & $\mathrm{RF}$ & CRF $2 \mathrm{~L} \not \mathrm{CRF}$ & $\mathrm{RF}$ & $\mathrm{CRF}$ & $2 \mathrm{~L}_{\xi} \mathrm{CRF}$ & $\mathrm{RF}$ & $\mathrm{CRF} 2 \mathrm{~L} z \mathrm{CRF}$ & $\mathrm{RF}$ & CRF 2L $2 \mathrm{CRF}$ & $\mathrm{RF}$ & CRF 2L $2 \mathrm{CRF}$ \\
\hline 16 & 80.3 & 81.8 & 85.1 & 82.6 & $\begin{array}{lll}5 & 84.8 & 87.5\end{array}$ & 0.72 & 0.75 & 0.79 & 0.76 & 0.790 .82 & 60.0 & $60.0 \quad 58.4$ & 63.7 & $63.3 \quad 58.9$ \\
\hline 17 & 77.7 & 79.2 & 83.4 & 82.9 & 83.386 .3 & 0.71 & 0.73 & 0.78 & 0.77 & 0.780 .82 & 59.8 & 60.662 .8 & 69.3 & 68.364 .5 \\
\hline 18 & 69.6 & 71.8 & 78.1 & 71.2 & 273.479 .9 & 0.57 & 0.6 & 0.68 & 0.58 & 0.610 .70 & 62.2 & 63.466 .9 & 61.2 & $62.5 \quad 65.9$ \\
\hline 19 & 67.4 & +68.6 & 69.5 & 65.5 & $\begin{array}{lll}5 & 66.8 & 69.4\end{array}$ & 0.57 & 0.59 & 0.60 & 0.55 & 0.570 .60 & 60.0 & $60.5 \quad 60.5$ & 68.7 & $70.3 \quad 62.2$ \\
\hline 20 & 76.2 & 77.7 & 82.9 & 78.0 & $79.2 \quad 84.4$ & 0.69 & 0.71 & 0.78 & 0.72 & 0.730 .80 & 65.6 & 67.071 .1 & 67.2 & 68.372 .3 \\
\hline Avg. ${ }^{\dagger}$ & 75.4 & 76.9 & $80.9(+4.0)$ & 77.3 & $\begin{array}{ll}78.8 & 82.7(+3.9)\end{array}$ & 0.69 & 0.71 & $0.76(+0.05)$ & 0.71 & $0.730 .78(+0.05)$ & 66.0 & $67.069 .5(+2.5)$ & 67.1 & $67.870 .5(+2.7)$ \\
\hline Ov.* & 74.2 & 75.8 & $79.8(+4.0)$ & 76.0 & $77.581 .5(+4.0)$ & 0.65 & 0.67 & $0.73(+0.06)$ & 0.68 & $0.700 .75(+0.05)$ & 61.5 & $62.363 .9(+1.6)$ & 66.0 & $66.664 .8(-1.8)$ \\
\hline
\end{tabular}

random forest, the $2 \mathrm{~L} \not \mathrm{CRF}$ approach allows to increase consistently the performances of the base independent models with average increases of accuracy of around 4\%. An exception is found when considering the mean of the average accuracy (AA) over the five tiles ('Ov.' in the table): in this case, the $1.8 \%$ decrease in AA observed is due to poor performance in tile \#16 (- 5\%). The poor performance is explained by the disappearance of the class 'bare soil' from the prediction, which decreases strongly the average accuracy. When pooling confusion matrices over the five tiles ('Avg.' in the table) this effect disappears, as bare soil is correctly predicted in the other tiles.

Random forests vs. CNNs. Table $\Pi$ presents the numerical results of the same dataset, this time obtained by a CNN predicting single labels per patch. The first striking observation is that all the figures of merit have a sharp improvement of about $10-15 \%$ in overall accuracy (OA), 10-18 $\kappa$ points or $8-10 \%$ in AA. Certainly the random forest results could have been improved by a more in depth research on feature engineering of the input space. However, considering that the CNN learns end-to-end, we only devoted efforts in finding the appropriate architecture training well. This is in line with observations in several other recent papers.

CNNs. Considering the role of the proposed $2 \mathrm{~L} \downarrow \mathrm{CRF}$ when using unary potentials from a CNN, we observe a general improvement of the accuracy figures, although it is less striking than in the previous case of random forests, in particular on pixels results. The reason lies in the high 
TABLE II

ZURICH SUMmER DATASET: NUMERICAL RESUltS ON THE FIVE TEST TILES. EACH PAIR OF COLUMNS ILLUSTRATES THE CHANGE OF PERFORMANCE BETWEEN THE UNARIES (CNN) AND THE SPATIAL MODEL USING THEM AS A BASE PROBABILISTIC INPUT IN THE UNARY POTENTIAL. THE CRF IS BASED ON [58].

\begin{tabular}{|c|c|c|c|c|c|c|c|c|c|c|c|c|c|}
\hline \multirow[b]{2}{*}{$\begin{array}{c}\text { Tile } \\
\#\end{array}$} & \multicolumn{4}{|c|}{ Ov. Accuracy (OA) } & \multicolumn{5}{|c|}{ Kappa $(\kappa)$} & \multicolumn{4}{|c|}{ Av. Accuracy (AA) } \\
\hline & $\mathrm{CNN}$ & $\begin{array}{l}\text { Pixels } \\
\text { CRF 2L } \downarrow \text { CRF }\end{array}$ & $\mathrm{CNN}$ & $\begin{array}{l}\text { Regions } \\
\text { CRF 2L々CRF }\end{array}$ & $\mathrm{CNN}$ & $\begin{array}{r}\text { Pixe } \\
\text { CRF } \\
\end{array}$ & $2 \mathrm{~L} \not \mathrm{CRF}$ & $\mathrm{CNN}$ & $\begin{array}{c}\text { Regions } \\
\text { CRF 2L々CRF }\end{array}$ & $\mathrm{CNN}$ & $\begin{array}{c}\text { Pixels } \\
\text { CRF 2L々CRF }\end{array}$ & $\mathrm{CNN}$ & $\begin{array}{l}\text { Regions } \\
\text { CRF 2L々CRF }\end{array}$ \\
\hline 16 & 90.7 & 90.791 .0 & 87.0 & 87.092 .3 & 0.87 & 0.87 & 0.87 & 0.82 & $0.82 \quad 0.89$ & 73.2 & 73.273 .2 & 69.1 & 69.078 .1 \\
\hline 17 & 90.2 & $90.2 \quad 90.4$ & 88.5 & 88.593 .0 & 0.87 & 0.87 & 0.87 & 0.85 & $\begin{array}{ll}0.85 & 0.91\end{array}$ & 69.5 & $69.4 \quad 69.5$ & 70.0 & 70.071 .9 \\
\hline 18 & 91.1 & 91.191 .2 & 90.7 & 90.792 .7 & 0.87 & 0.87 & 0.87 & 0.86 & $\begin{array}{ll}0.86 & 0.89\end{array}$ & 86.9 & 86.987 .1 & 85.3 & 85.388 .0 \\
\hline 19 & 90.4 & 90.490 .4 & 87.7 & 87.791 .4 & 0.87 & 0.87 & 0.87 & 0.84 & $\begin{array}{lll}0.84 & 0.89\end{array}$ & 91.1 & 91.191 .2 & 87.2 & 87.292 .9 \\
\hline 20 & 89.6 & 89.789 .9 & 88.0 & 88.091 .1 & 0.86 & 0.86 & 0.87 & 0.84 & $\begin{array}{lll}0.84 & 0.89\end{array}$ & 76.0 & 76.076 .2 & 74.1 & 74.177 .3 \\
\hline Avg. ${ }^{\dagger}$ & 90.3 & $90.390 .5(+0.2)$ & 88.1 & $88.192 .0(+3.9)$ & 0.88 & 0.88 & 0.88 & 0.85 & $0.850 .90(+0.05)$ & 77.7 & $77.777 .8(+0.1)$ & 76.5 & $76.579 .3(+2.8)$ \\
\hline Ov.* & 90.4 & $90.490 .6(+0.2)$ & 88.3 & $88.492 .1(+3.7)$ & 0.87 & 0.87 & 0.87 & 0.84 & $0.840 .89(+0.05)$ & 79.3 & 79.479 .4 & 77.1 & $77.181 .6(+4.5)$ \\
\hline
\end{tabular}

accuracy of the initial result, for which the unary scores are sharp and spatially consistent (see the third column of Fig. 6). This can be seen in the difference between the CNN results and those of the CRF based on [58], which considers only spatial interactions for each lattice separately. The $2 \mathrm{~L}_{\downarrow} \mathrm{CRF}$ model only needs to correct for inconsistent labelings among the two layers, which results in small increases in the accuracies. On the contrary, the high accuracy and consistency of the unary scores allows to correct for several misclassifications at the region level, for which the pooling of the unary scores resulted in erroneous region scores, in particular in the case of regions with high entropy of the posterior probability at the pixel level. For instance, the road network is poorly reconstructed in tile \#16 in Fig. 6, as regions composing it are often incorrectly classified as buildings. On the contrary, they are assigned to the roads class by $2 \mathrm{~L} \downarrow \mathrm{CRF}$, since the evidence from the pixel representation is strong enough to influence the response at the region level, thus leading to a map that is correct (by the pixel response) and sharp (by the region topology). Another example is in tile \#19, where the river is correctly recovered by $2 \mathrm{~L} \downarrow \mathrm{CRF}$, while the original region results were providing a mix between water, trees, and roads.

Parameters analysis. Figure 7 reports the sensitivity analysis for the $\mu$ and $\lambda$ parameters, involved in Eq. (6). As a reminder, $\mu$ control the strength of the relations between the spatial supports and $\lambda$ the strength of the local spatial smoothing within each spatial support. In all experiments, the model not making any use of inter-support connections $(\mu=0$, which would 


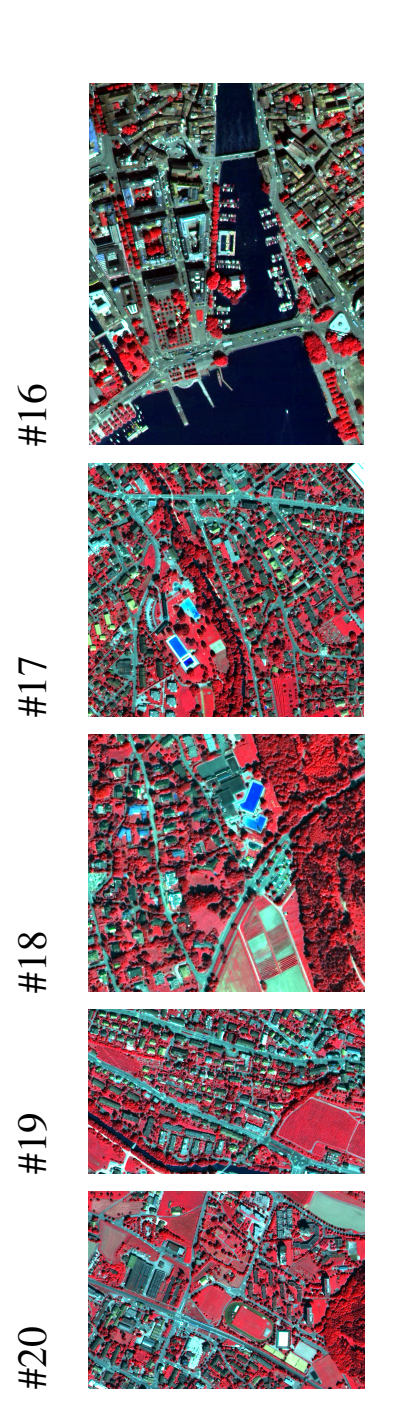

(a) image
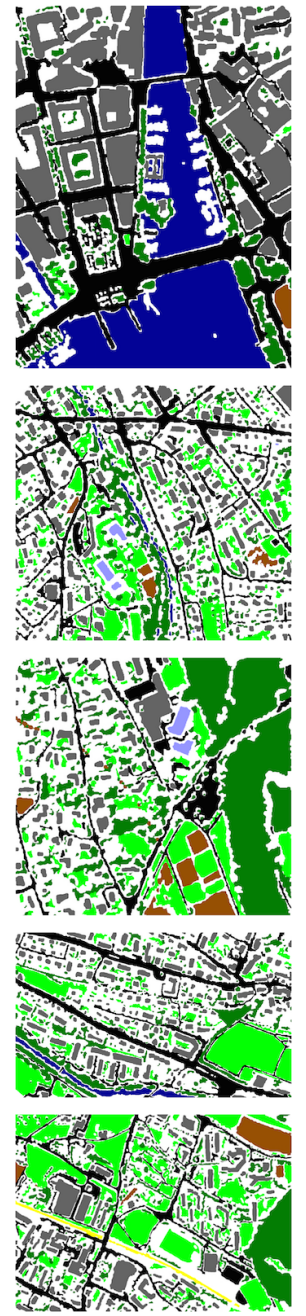

(b) ground truth
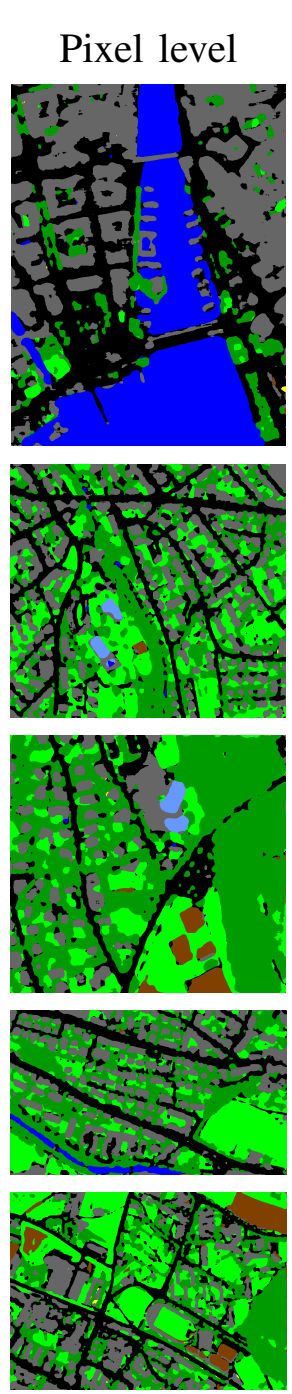

(c) $\mathrm{CNN}$
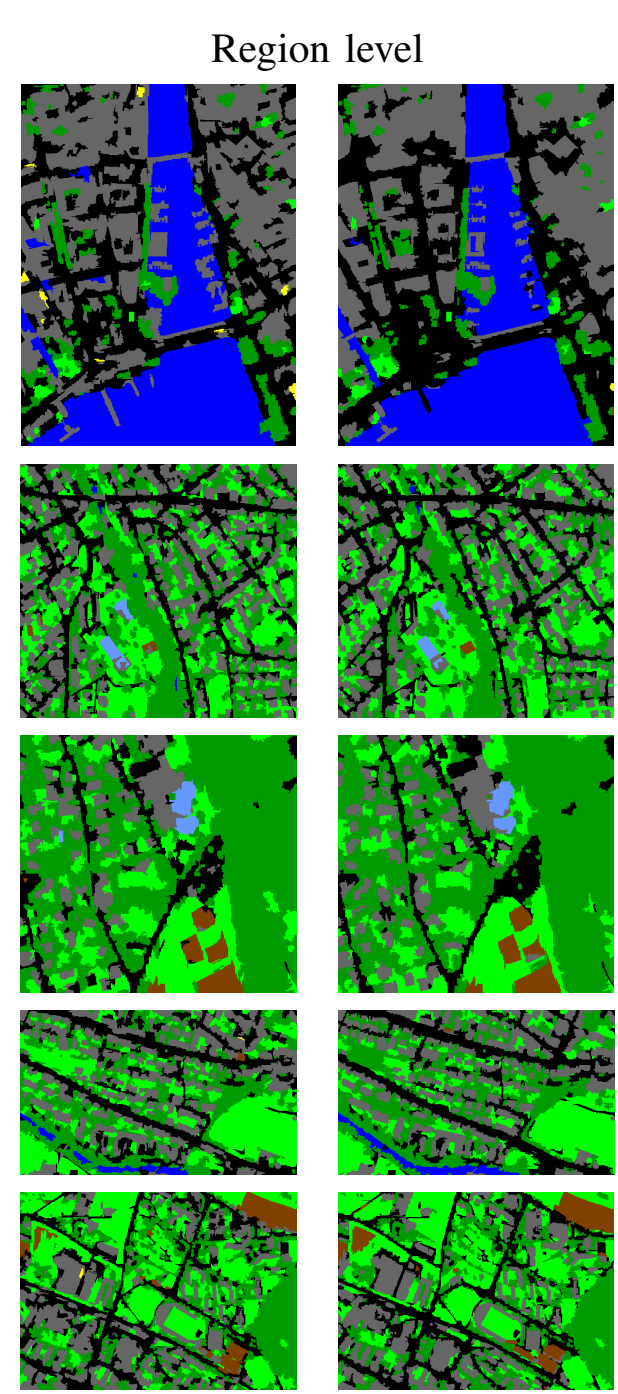

(d) $\mathrm{CNN}$
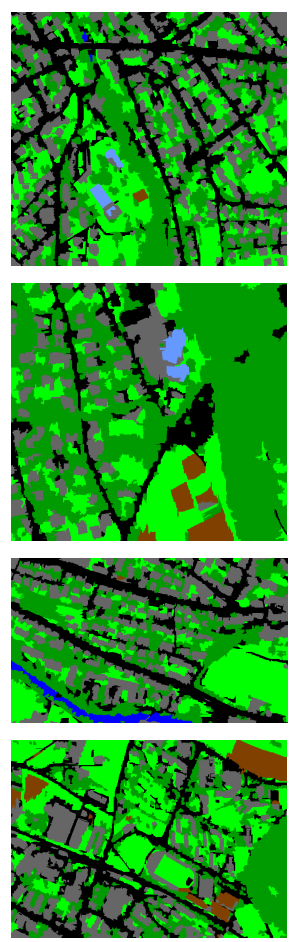

(e) $2 \mathrm{~L} \not \mathrm{CRF}$

Fig. 6. Zurich Summer dataset: results on the five test images. (a) original image; (b) ground truth; (c) CNN, pixel-based;

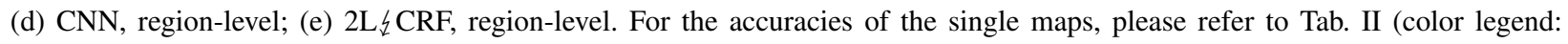
residential, street, trees, meadows, railway, water, swimming pools, bare soil).

roughly correspond to optimizing two separate CRFs) does not provide the best results, thus showing the interest of a joint model. This is particularly visible at the region level, where the evidence from the pixel level is able to successfully revert many errors, in particular for the RF classifier, which often provides ambiguous class-likelihoods. Also, using contrast sensitivity seems particularly important at the pixel level, while less at the region level: this is expected, since given the high resolution of the data, local inconsistencies at the pixel level are more frequent that noisy predictions at the region level. 

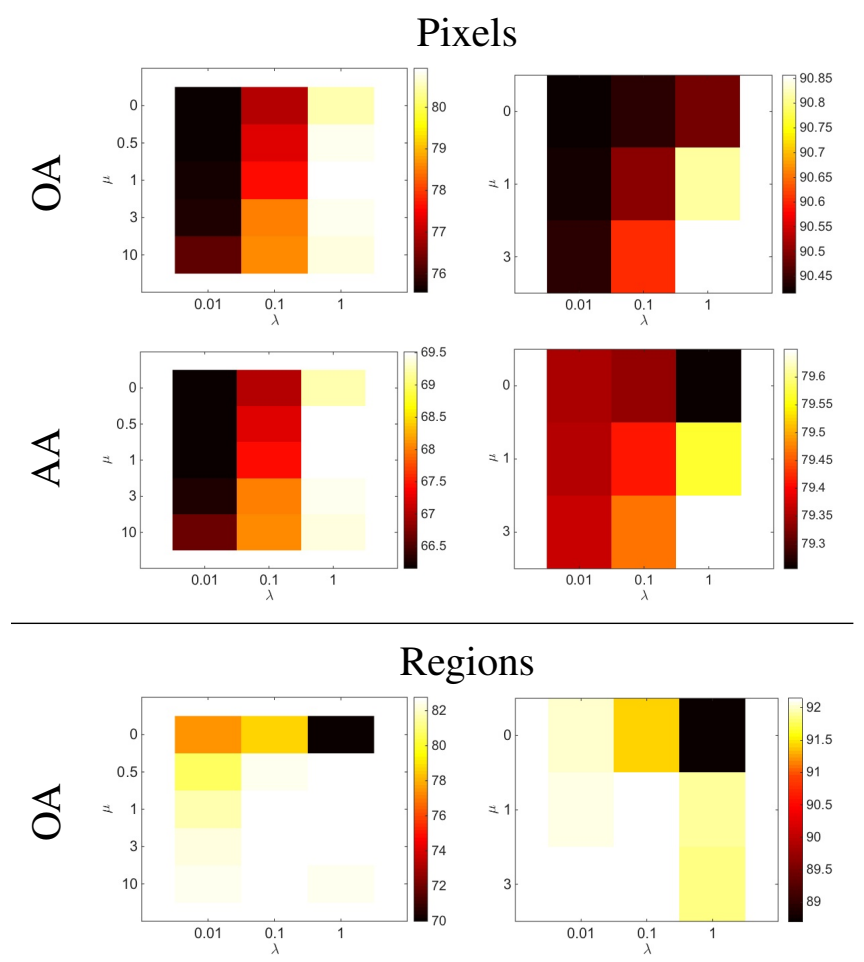

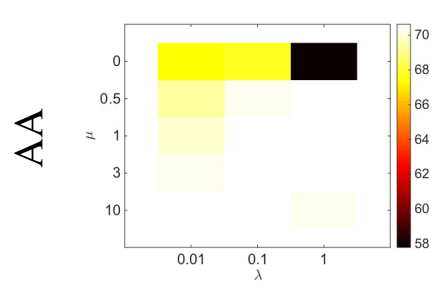

$\mathrm{RF}$

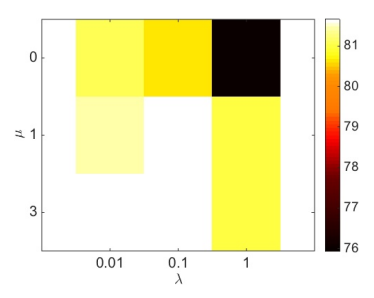

CNN

Fig. 7. Sensibility analysis for the $\mu$ and $\lambda$ parameters in Eq. 6. and the Zurich Summer dataset.

\section{B. Zeebruges dataset}

In the case of the Zeebruges dataset, we retrieve unary scores directly from the model of [31]. This model achieves results that compare favorably to the state-of-the-art methods on this dataset.It still suffers from some prediction noise leading to small artifacts, as it can be seen in the maps in Fig. 8 for the entire tiles and in Fig. 9 for details. This is certainly due to the increased spatial resolution (and therefore complexity) of the problem, since we are working, compared to the Zurich Summer dataset, on a ten times higher spatial resolution and without an infrared channel.

Numerical results are reported in Tab. III; as a first observation, the standard CRF approach seems to improve the results only marginally, which can be explained by two factors: first, 
TABLE III

NUMERICAL RESUlTS ON THE ZEEBRUGES DATASET (GRSS_DFC_2015). EACH PAIR OF COLUMNS ILLUSTRATES THE CHANGE OF PERFORMANCE BETWEEN THE UNARIES (CNN) AND THE SPATIAL MODEL USING THEM AS A BASE IN THE UNARY POTENTIAL. THE CRF IS BASED ON [58].

\begin{tabular}{|c|c|c|c|c|c|c|c|c|c|c|c|c|}
\hline \multirow[b]{2}{*}{$\begin{array}{c}\text { Tile } \\
\#\end{array}$} & \multicolumn{4}{|c|}{ Ov. Accuracy (OA) } & \multicolumn{4}{|c|}{ Kappa $(\kappa)$} & \multicolumn{4}{|c|}{ Av. Accuracy (AA) } \\
\hline & $\mathrm{CNN}$ & $\begin{array}{l}\text { Pixels } \\
\text { CRF 2L々CRF }\end{array}$ & $\mathrm{CNN}$ & $\begin{array}{l}\text { Regions } \\
\text { CRF 2L々CRF }\end{array}$ & $\mathrm{CNN}$ & $\begin{array}{c}\text { Pixels } \\
\text { CRF 2L々CRF }\end{array}$ & $\mathrm{CNN}$ & $\begin{array}{l}\text { Regions } \\
\text { CRF 2L } 2 \mathrm{CRF}\end{array}$ & $\mathrm{CNN}$ & $\begin{array}{l}\text { Pixels } \\
\text { CRF 2L々CRF }\end{array}$ & $\mathrm{CNN}$ & $\begin{array}{l}\text { Regions } \\
\text { CRF 2L々CRF }\end{array}$ \\
\hline Tile \#6 & 77.8 & 78.078 .9 & 79.3 & 79.680 .0 & 0.68 & 0.680 .69 & 0.70 & $\begin{array}{ll}0.70 & 0.71\end{array}$ & 65.2 & 65.265 .4 & 65.9 & 65.966 .2 \\
\hline Avg..$^{\dagger}$ & 82.6 & $82.783 .3(+0.6)$ & 83.3 & $83.583 .7(+0.2)$ & 0.77 & $0.77 \quad 0.78(+0.01)$ & 0.78 & 0.790 .79 & 75.2 & $75.375 .6(+0.3)$ & 76.4 & $76.476 .5(+0.1)$ \\
\hline
\end{tabular}

and as for the previous dataset, the CNN unaries are very sharp and confident (even when misclassifying), making the change of a label very unlikely. Secondly, the use of CRFs with up to pairwise nonzero clique potentials rather than more sophisticated higher order CRFs (which was necessary to keep the problem computationally feasible) made label swaps improbable, since the pixel classifier was not much affected by salt and pepper noise, but rather by larger spatial artifacts that could not be corrected by looking only at the direct pixel neighborhood. This is the reason why, for this dataset, $2 \mathrm{~L} \downarrow \mathrm{CRF}$ greatly increases performance at the pixel level: by letting the pixel lattice be influenced by the region structure, where the scores are pooled per region, the pixel CNN becomes aware of larger neighborhood structures and can therefore correct for larger confident misclassifications at pixel level (see the zooms in Fig. 9 for some examples). Such beneficial effect could have been maybe reinforced if the region level CNN were a classifier trained specifically to predict land cover at the region level (and not a set of region-pooled scores over the pixel unaries), but this would have implied training a separate CNN model for the region scale. The consequent significant increase in memory and computational resources would diminish - in our opinion - the interest of the combined approach.

\section{CONCLUSiON}

In this paper, we proposed a probabilistic discriminative graphical model relying on a conditional random fields formulation for the fusion of land-cover and land-use classification results from very high resolution remote sensing images. The system is able to find agreement between 


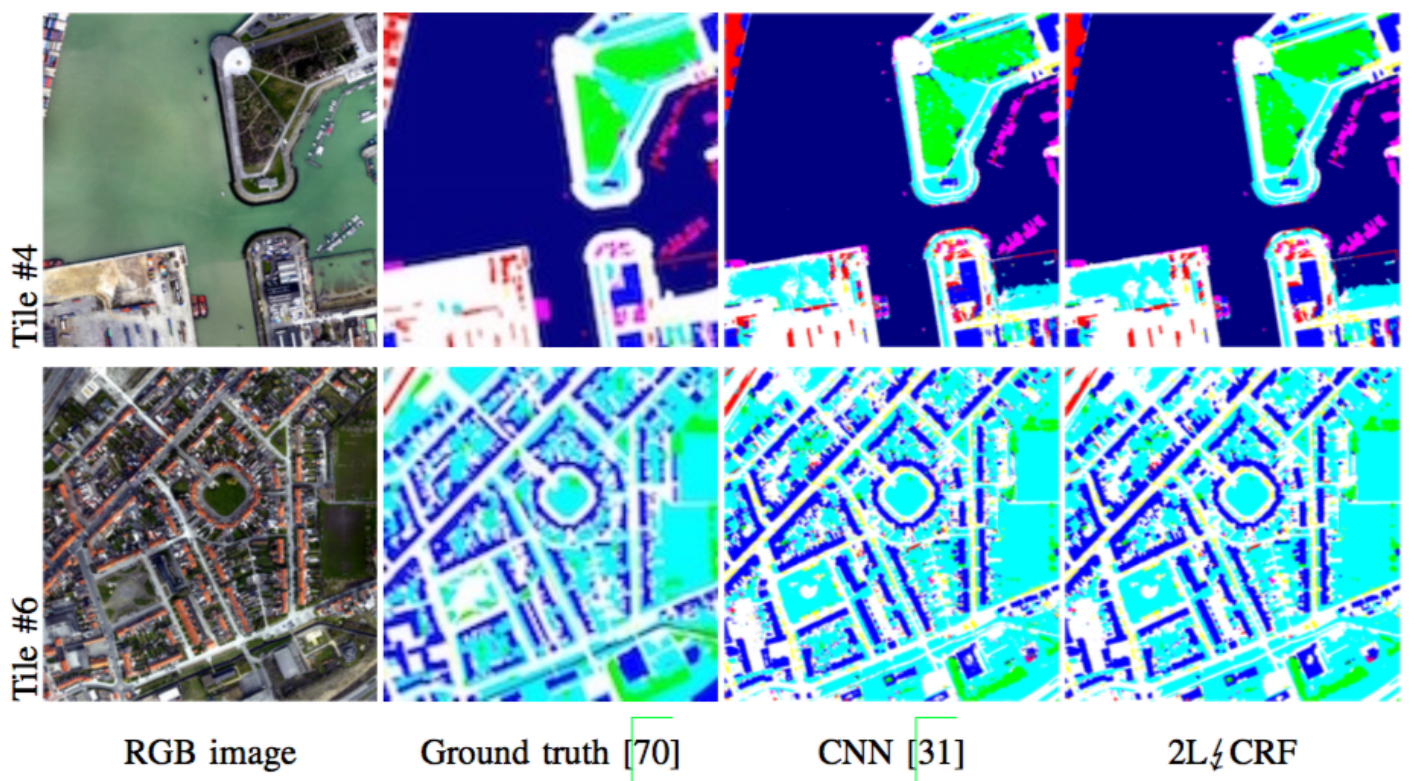

Fig. 8. Zeebruges dataset (grss_dfc_2015): results on the two test tiles. (a) original image; (b) ground truth; (c) CNN, pixel-based; (d) $2 \mathrm{~L} \downarrow \mathrm{CRF}$, pixel level. For the accuracies of the single maps, please refer to Tab. III] (color legend: impervious (white colored), water, clutter, low vegetation, buildings, trees, boats, cars). The ground truth images are blurred as in [3], since they are undisclosed. (Note: in this preprint we had to decrease the graphics resolution. For full resolution, please refer to the published version, or contact the authors).

probabilistic decisions with multiple spatial supports. We explored the fusion of pixel- and region-based spatial supports within an energy minimization framework, where each individual classification result is tributary of i) the posterior distribution of the land cover classes at the single instance level (the pixel or the region), ii) the spatial smoothness of the predictions (i.e. the consistency of the prediction among the spatial neighbors), and iii) the smoothness across supports (i.e. the consistency of the predictions between a region and the pixels composing the region itself). These three goals are addressed jointly within a conditional random field model with connections across layers corresponding to different spatial support representations. It is also proven that the proposed two-layer model can be flattened into a single CRF, for which energy minimization can be addressed efficiently with standard energy minimization solvers.

Applications to two very high resolution benchmark datasets showed the potential of the approach that, using common models (we considered random forests and convolutional neural networks), can improve the final maps consistently and joining the spatial detail of the pixel support with the geometrical object accuracy of the region support. In the future, we would 

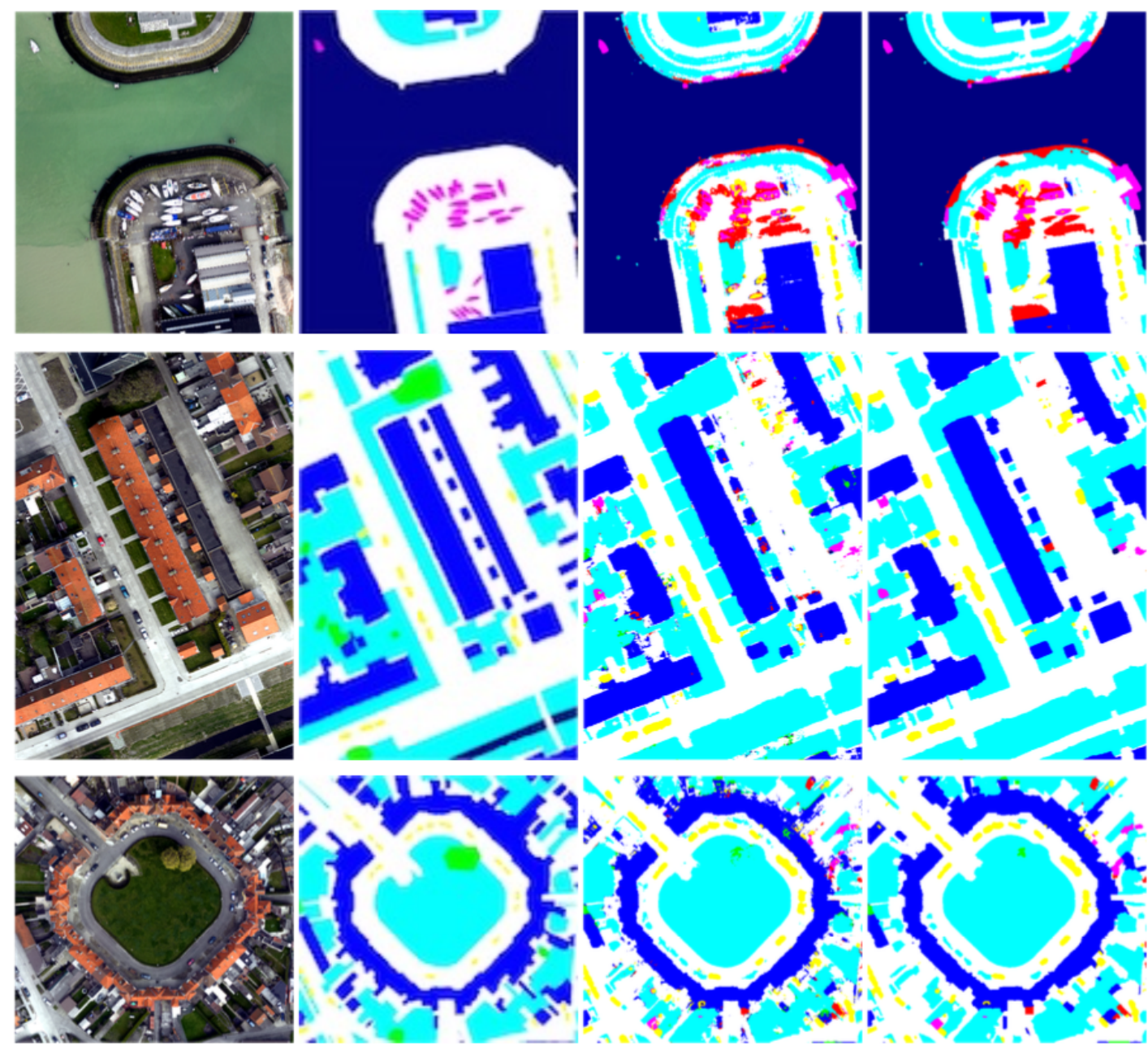

RGB image

Ground truth [70]

CNN [31]

$2 \mathrm{~L} \not \mathrm{CRF}$

Fig. 9. Zeebruges dataset (grss_dfc_2015): zoomed results on details of the two test tiles of Fig. 8 (a) original image; (b) ground truth; (c) CNN, pixel-based; (d) $2 \mathrm{~L} \downarrow \mathrm{CRF}$, pixel level. For the accuracies of the single maps, please refer to Tab. III (color legend: impervious (white coloured), water, clutter, low vegetation, buildings, trees, boats, cars). The ground truth images are blurred as in [3], since they are undisclosed. (Note: in this preprint we had to decrease the graphics resolution. For full resolution, please refer to the published version, or contact the authors).

like to test the $2 \mathrm{~L} \downarrow \mathrm{CRF}$ model for the fusion of multitemporal data, multiple segmentations $(>2)$, or for applications involving different classes to be predicted for different spatial supports. Furthermore, although the impact of the weight parameters of the proposed CRF model on the resulting performance was limited, it would also be interesting to automatically optimize their values, for example using log-likelihood-like (e.g. through pseudo-likelihood approximations or the expectation-maximization algorithm) or mean-square-error concepts [35], [74], [75]. 


\section{ACKNOWLEDGMENTS}

This work was supported in part by the Swiss National Science Foundation, via the grant 150593 "Multimodal machine learning for remote sensing information fusion" (http://p3.snf.ch/project150593). The authors would also like to thank the Belgian Royal Military Academy, for acquiring and providing the Zeebruges data used in this study, ONERA - The French Aerospace Lab, for providing the corresponding ground-truth data [70], and the IEEE GRSS Image Analysis and Data Fusion Technical Committee.

\section{REFERENCES}

[1] J. Berni, P. Zarco-Tejada, L. Suarez, and E. Fereres, "Thermal and narrowband multispectral remote sensing for vegetation monitoring from an unmanned aerial vehicle," IEEE Trans. Geosci. Remote Sens., vol. 47, no. 3, pp. 722-738, 2009.

[2] R. Zahawi, J. Dandois, K. Holl, D. Nadwodny, J. Reid, and E. Ellis, "Using lightweight unmanned aerial vehicles to monitor tropical forest recovery," Biological Conservation, vol. 186, pp. 287-295, 2015.

[3] M. Campos-Taberner, A. Romero-Soriano, C. Gatta, G. Camps-Valls, A. Lagrange, B. L. Saux, A. Beaupère, A. Boulch, A. Chan-Hon-Tong, S. Herbin, H. Randrianarivo, M. Ferecatu, M. Shimoni, G. Moser, and D. Tuia, "Processing of extremely high resolution LiDAR and RGB data: Outcome of the 2015 IEEE GRSS Data Fusion Contest. Part A: 2D contest," IEEE J. Sel. Topics Appl. Earth Observ. Remote Sens., vol. 9, no. 12, pp. 5547-5559, 2016.

[4] T. Tomic, K. Schmid, P. Lutz, A. Domel, M. Kassecker, E. Mair, I. Grixa, F. Ruess, M. Suppa, and D. Burschka, "Toward a fully autonomous uav: Research platform for indoor and outdoor urban search and rescue," IEEE Robotics and Automation Magazine, vol. 19, no. 3, pp. 46-56, 2012.

[5] T. Blaschke, "Object based image analysis for remote sensing," ISPRS J. Int. Soc. Photo. Remote Sens., vol. 65, no. 1, pp. $2-16,2010$.

[6] P. Felzenszwalb and D. Huttenlocher, "Efficient graph-based image segmentation,” Int. J. Comp. Vision, 2004.

[7] M.-Y. Liu, O. Tuzel, S. Ramalingam, and R. Chellappa, "Entropy rate superpixel segmentation," in Proc. CVPR, 2011, pp. 2097-2104.

[8] R. Achanta, A. Shaji, K. Smith, A. Lucchi, P. Fua, and S. SÃ $\frac{1}{4}$ sstrunk, "Slic superpixels compared to state-of-the-art superpixel methods," IEEE Trans. Pattern Anal. Mach. Intell., vol. 34, no. 11, pp. 2274-2281, 2012.

[9] M. Dalla Mura, J. Atli Benediktsson, B. Waske, and L. Bruzzone, "Morphological attribute profiles for the analysis of very high resolution images," IEEE Trans. Geosci. Remote Sens., vol. 48, no. 10, pp. 3747-3762, 2010.

[10] F. Pacifici, M. Chini, and W. Emery, "A neural network approach using multi-scale textural metrics from very highresolution panchromatic imagery for urban land-use classification,” Remote Sens. Environ., vol. 113, no. 6, pp. 1276-1292, 2009.

[11] L. Huang, C. Chen, W. Li, and Q. Du, "Remote sensing image scene classification using multi-scale completed local binary patterns and fisher vectors," Remote Sens., vol. 8, no. 6, 2016.

[12] U. Budak, U. Halici, A. Sengur, M. Karabatak, and Y. Xiao, "Efficient airport detection using line segment detector and fisher vector representation," IEEE Geosci. Remote Sens. Letters, vol. 13, no. 8, pp. 1079-1083, 2016.

[13] Q. Zhu, Y. Zhong, B. Zhao, G.-S. Xia, and L. Zhang, "Bag-of-visual-words scene classifier with local and global features for high spatial resolution remote sensing imagery," IEEE Geosci. Remote Sens. Letters, vol. 13, no. 6, pp. 747-751, 2016.

[14] J. Tu, H. Sui, W. Feng, K. Sun, and L. Hua, "Detection of damaged rooftop areas from high-resolution aerial images based on visual bag-of-words model," IEEE Geosci. Remote Sens. Letters, vol. 13, no. 12, pp. 1817-1821, 2016. 
[15] N. Rey, M. Volpi, S. Joost, and D. Tuia, "Detecting animals in african savanna with UAVs and the crowds," Remote Sens. Environ., in press.

[16] M. Volpi and V. Ferrari, "Structured prediction for urban scene semantic segmentation with geographic context," in Proc. JURSE, Lausanne, Switzerland., 2015.

[17] D. Konstantinidis, T. Stathaki, V. Argyriou, and N. Grammalidis, "Building detection using enhanced hog-lbp features and region refinement processes,” IEEE J. Sel. Topics Appl. Earth Observ. Remote Sens., vol. PP, no. 99, 2016.

[18] D. Tuia, M. Volpi, M. dalla Mura, A. Rakotomamonjy, and R. Flamary, "Automatic feature learning for spatio-spectral image classification with sparse SVM," IEEE Trans. Geosci. Remote Sens., vol. 52, no. 10, pp. 6062-6074, 2014.

[19] D. Tuia, N. Courty, and R. Flamary, "Multiclass feature learning for hyperspectral image classification: sparse and hierarchical solutions," ISPRS J. Int. Soc. Photo. Remote Sens., vol. 105, pp. 272-285, 2015.

[20] M. Volpi and V. Ferrari, "Semantic segmentation of urban scenes by learning local class interactions," in Proc. CVPR workshops, 2015.

[21] Y. LeCun, L. Bottou, Y. Bengio, and P. Haffner, "Gradient-based learning applied to document recognition," Proc. IEEE, 1998.

[22] M. Volpi and D. Tuia, "Dense semantic labeling of subdecimeter resolution images with convolutional neural networks," IEEE Trans. Geosci. Remote Sens., vol. 55, no. 2, pp. 881-893, 2017.

[23] X. Zhu, D. Tuia, L. Mou, G. Xia, L. Zhang, F. Xu, and F. Fraundorfer, "Deep learning in remote sensing: a review,” IEEE Geosci. Remote Sens. Mag., in press.

[24] J. Long, E. Shelhamer, and T. Darrell, "Fully convolutional networks for semantic segmentation," in Proc. CVPR workshops, 2015.

[25] B. Hariharan, P. A. Arbeláez, R. B. Girshick, and J. Malik, "Hypercolumns for object segmentation and fine-grained localization," in Proc. CVPR, vol. abs/1411.5752, 2015.

[26] V. Badrinarayanan, A. Kendall, and R. Cipolla, "Segnet: A deep convolutional encoder-decoder architecture for image segmentation," IEEE Trans. Pattern Anal. Mach. Intell., 2017.

[27] N. Audebert, B. Le Saux, and S. Lefèvre, "Semantic segmentation of earth observation data using multimodal and multiscale deep networks," in Proc. ACCV, 2016.

[28] D. Marmanis, K. Schindler, J. D. Wegner, S. Galliani, M. Datcu, and U. Stilla, "Classification with an edge: Improving semantic image segmentation with boundary detection," arXiv:1612.01337, 2016.

[29] E. Maggiori, Y. Tarabalka, G. Charpiat, and P. Alliez, "Convolutional neural networks for large-scale remote-sensing image classification,” IEEE Trans. Geosci. Remote Sens., vol. 55, no. 2, pp. 645-657, 2017.

[30] — - "High-resolution aerial image labeling with convolutional neural networks," arXiv: 1611.01962, 2016.

[31] D. Marcos, M. Volpi, and D. Tuia, "Land cover mapping at very high resolution with rotation equivariant CNNs: towards small yet accurate models," ISPRS J. Int. Soc. Photo. Remote Sens., submitted.

[32] J. Sherrah, "Fully convolutional networks for dense semantic labelling of high-resolution aerial imagery," arXiv:1606.02585, 2016.

[33] S. Geman and D. Geman, "Stochastic relaxation, Gibbs distributions, and the Bayesian restoration of images," IEEE Trans. Pattern Anal. Mach. Intell., vol. PAMI-6, no. 6, pp. 721-741, 1984.

[34] J. Besag, "Statistical analysis of dirty pictures," Journal of Applied Statistics, vol. 20, no. 5-6, pp. 63-87, 1993.

[35] Z. Kato and J. Zerubia, "Markov random fields in image segmentation," Foundations and Trends in Signal Processing, vol. 5, no. 1-2, pp. 1-155, 2012.

[36] J. Lafferty, A. McCallum, and F. Pereira, "Conditional random fields: Probabilistic models for segmenting and labeling sequence data," in ICML, 2001, pp. 282-289. 
[37] C. Sutton and A. McCallum, "An introduction to conditional random fields," Foundations and Trends in Machine Learning, vol. 4, no. 4, pp. 267-373, 2011.

[38] G. Moser, S. B. Serpico, and J. A. Benediktsson, "Land-cover mapping by Markov modeling of spatial-contextual information," Proc. IEEE, vol. 101, no. 3, pp. 631-651, 2013.

[39] K. Schindler, "An overview and comparison of smooth labeling methods for land-cover classification," IEEE Trans. Geosci. Remote Sens., vol. 50, no. 11, pp. 4534-4545, 2012.

[40] S. Li, Markov random field modeling in image analysis. Springer, 2009.

[41] P. C. Smits and S. Dellepiane, "Synthetic aperture radar image segmentation by a detail preserving Markov random field approach,” IEEE Trans. Geosci. Remote Sens., vol. 35, no. 4, pp. 844-857, 1997.

[42] C. Luo and G. Sohn, "Scene-layout compatible conditional random field for classifying terrestrial laser point clouds," in ISPRS Annals of Photogrammetry, Remote Sensing and Spatial Information Sciences, vol. II-3, 2014, pp. 79-86.

[43] A. Solberg, T. Taxt, and A. Jain, "A Markov random field model for classification of multisource satellite imagery," IEEE Trans. Geosci. Remote Sens., vol. 34, no. 1, pp. 100-113, 1996.

[44] D. Marcos, R. Hamid, and D. Tuia, "Geospatial correspondence for multimodal registration," in Proc. CVPR, Las Vegas, NV, 2016.

[45] T. Hoberg, F. Rottensteiner, R. Q. Feitosa, and C. Heipke, "Conditional random fields for multitemporal and multiscale classification of optical satellite imagery," IEEE Trans. Geosci. Remote Sens., vol. 53, no. 2, pp. 659-673, 2015.

[46] J. D. Wegner, S. Branson, D. Hall, K. Schindler, and P. Perona, "Cataloguing public objects using aerial and street-level images - urban trees," in Proc. CVPR, Las Vegas, NV, 2016.

[47] A. Willsky, "Multiresolution Markov models for signal and image processing," Proc. IEEE, vol. 90, no. 8, pp. 1396-1458, 2002.

[48] G. Scarpa, R. Gaetano, M. Haindl, and J. Zerubia, "Hierarchical multiple Markov chain model for unsupervised texture segmentation,” IEEE Trans. Image Proc., vol. 18, no. 8, pp. 1830-1843, 2009.

[49] G. Moser, A. De Giorgi, and S. Serpico, "Multiresolution supervised classification of panchromatic and multispectral images by Markov random fields and graph cuts," IEEE Trans. Geosci. Remote Sens., vol. 43, no. 8, pp. 1901-1911, 2016.

[50] I. Hedhli, G. Moser, S. Serpico, and J. Zerubia, "A new cascade model for the hierarchical joint classification of multitemporal and multiresolution remote sensing data," IEEE Trans. Geosci. Remote Sens., vol. 54, no. 11, pp. 6333-6348, 2016.

[51] P. Kohli, L. Ladicky, and P. H. Torr, "Robust higher order potentials for enforcing label consistency," in Proc. CVPR, 2008.

[52] J. Wegner, J. Montoya-Zegarra, and K. Schindler, "Road networks as collections of minimum cost paths," ISPRS J. Int. Soc. Photo. Remote Sens., vol. 108, pp. 128-137, 2015.

[53] L. Albert, F. Rottensteiner, and C. Heipke, "A higher order conditional random field model for simultaneous classification of land cover and land use," ISPRS J. Int. Soc. Photo. Remote Sens., vol. 130, pp. 63-80, 2017.

[54] C. Rother, V. Kolmogorov, T. A. Minka, and A. Blake, "Co-segmentation of image pairs by histogram matching incorporating a global constraint into MRFs," in Proc. CVPR, 2006.

[55] A. Joulin, F. Bach, and J. Ponce, "Discriminative clustering for image co-segmentation,” in Proc. CVPR, 2010.

[56] P. Xiao, M. Yuan, X. Zhang, X. Feng, and Y. Guo, "Co-segmentation for object-based building change detection from high-resolution remotely sensed images,” IEEE Trans. Geosci. Remote Sens., pp. 1587-1603, 2017.

[57] Y. Huang, M. S. Brown, and D. Xu, "A framework for reducing ink-bleed in old documents," in Proc. CVPR, Anchorage, AK, 2008.

[58] Y. Boykov, O. Veksler, and R. Zabih, "Fast approximate energy minimization via graph cuts," IEEE Trans. Pattern Anal. Mach. Intell., vol. 23, no. 11, pp. 1222-1239, 2001. 
[59] G. Roig, X. Boix, H. B. Shitrit, and P. Fua, "Conditional random fields for multi-camera object detection," in Proc. ICCV, 2011, pp. 563-570.

[60] D. Tuia, M. Volpi, and G. Moser, "Getting pixels and regions to agree with conditional random fields," in IEEE International Geoscience and Remote Sensing Symposium, IGARSS, Beijing, China, 2016.

[61] F. Melgani and S. Serpico, "A Markov random field approach to spatio-temporal contextual image classification," IEEE Trans. Geosci. Remote Sens., vol. 41, no. 11, pp. 2478-2487, 2003.

[62] P. H. Swain, "Bayesian classification in a time-varying environment," IEEE Trans Syst Man Cybern, vol. SMC-8, no. 12, pp. 879-883, 1978.

[63] C. Bishop, Pattern Recognition and Machine Learning. Springer, 2006.

[64] L. Breiman, "Random forests," Machine Learning, vol. 45, no. 1, pp. 5-32, 2001.

[65] V. Vapnik, The Nature of Statistical Learning Theory. Springer, 2000.

[66] T.-F. Wu, C.-J. Lin, and R. Weng, "Probability estimates for multi-class classification by pairwise coupling," Journal of Machine Learning Research, vol. 5, pp. 975-1005, 2004.

[67] M. J. Wainwright and M. I. Jordan, "Graphical models, exponential families, and variational inference," Foundations and Trends in Machine Learning, vol. 1, no. 1-2, pp. 1-305, 2008.

[68] R. Szeliski, R. Zabih, D. Scharstein, O. Veksler, V. Kolmogorov, A. Agarwala, M. Tappen, and C. Rother, "A comparative study of energy minimization methods for Markov Random Fields," in Proc. ECCV, 2006.

[69] V. Kolmogorov, "Convergent tree-reweighted message passing for energy minimization," IEEE Trans. Pattern Anal. Mach. Intell., vol. 28, no. 10, pp. 1568-1583, 2006.

[70] A. Lagrange, B. L. Saux, A. Beaupere, A. Boulch, A. Chan-Hon-Tong, S. Herbin, H. Randrianarivo, and M. Ferecatu, "Benchmarking classification of earth-observation data: from learning explicit features to convolutional networks," in IEEE International Geoscience and Remote Sensing Symposium (IGARSS), 2015.

[71] K. Simonyan and A. Zisserman, "Very deep convolutional networks for large-scale image recognition," in Proc. ICLR, vol. abs/1409.1, 2015.

[72] N. Srivastava, G. Hinton, A. Krizhevsky, I. Sutskever, and R. Salakhutdinov, "Dropout: A simple way to prevent neural networks from overfitting," J. Mach. Learn. Res., vol. 15, no. 1, pp. 1929-1958, 2014.

[73] D. Marcos, M. Volpi, N. Komodakis, and D. Tuia, "RotEqNet: rotation equivariant vector field networks," in Proc. ICCV, Venezia, Italy, 2017.

[74] J. Gimenez, A. Frery, and A. Flesia, "When data do not bring information: A case study in Markov random fields estimation,” IEEE J. Sel. Topics Appl. Earth Observ. Remote Sens., vol. 8, no. 1, pp. 195-203, 2015.

[75] S. Serpico and G. Moser, "Weight parameter optimization by the Ho-Kashyap algorithm in MRF models for supervised image classification,” IEEE Trans. Geosci. Remote Sens., vol. 44, no. 12, pp. 3695-3705, 2006. 